\title{
A Continuum of Accretion Burst Behavior in Young Stars Observed by $K 2$
}

\author{
Ann Marie Cody $^{1}$, Lynne A. Hillenbrand ${ }^{2}$, Trevor J. David ${ }^{2}$, John M. Carpenter ${ }^{2,4}$, Mark E. Everett ${ }^{3}$, and Steve B. Howell ${ }^{1}$ \\ ${ }^{1}$ NASA Ames Research Center, Moffett Field, CA 94035, USA \\ ${ }^{2}$ Department of Astronomy, California Institute of Technology, Pasadena, CA 91125, USA \\ ${ }^{3}$ National Optical Astronomy Observatory, 950 N. Cherry Avenue, Tucson, AZ 85719, USA \\ Received 2016 September 2; revised 2016 December 15; accepted 2016 December 20; published 2017 February 7
}

\begin{abstract}
We present 29 likely members of the young $\rho$ Oph or Upper Sco regions of recent star formation that exhibit "accretion burst" type light curves in $K 2$ time series photometry. The bursters were identified by visual examination of their $\sim 80$-day light curves, though all satisfy the $M<-0.25$ flux asymmetry criterion for burst behavior defined by Cody et al. The burst sources represent $\approx 9 \%$ of cluster members with strong infrared excess indicative of circumstellar material. Higher amplitude burster behavior is correlated with larger inner disk infrared excesses, as inferred from WISE W1 - W2 color. The burst sources are also outliers in their large $\mathrm{H} \alpha$ emission equivalent widths. No distinction between bursters and non-bursters is seen in stellar properties such as multiplicity or spectral type. The frequency of bursters is similar between the younger, more compact $\rho$ Oph region, and the older, more dispersed Upper Sco region. The bursts exhibit a range of shapes, amplitudes $(\sim 10 \%-700 \%)$, durations $(\sim 1-10$ days), repeat timescales $(\sim 3-80$ days), and duty cycles $(\sim 10 \%-100 \%)$. Our results provide important input to models of magnetospheric accretion, in particular, by elucidating the properties of accretionrelated variability in the low state between major longer duration events such as EX Lup and FU Ori type accretion outbursts. We demonstrate the broad continuum of accretion burst behavior in young stars-extending the phenomenon to lower amplitudes and shorter timescales than traditionally considered in the theory of pre-main sequence accretion history.
\end{abstract}

Key words: accretion, accretion disks - protoplanetary disks - stars: pre-main sequence - stars: variables: T Tauri, Herbig $\mathrm{Ae} / \mathrm{Be}$ - techniques: photometric

Supporting material: extended figure

\section{Introduction}

Variable mass flux has long been recognized as an important element of protostellar and pre-main-sequence accretion. Accretion rates are believed to be higher $\left(\sim 10^{-5} M_{\odot} \mathrm{yr}^{-1}\right)$ during the first $10^{5}$ years of protostellar evolution, with frequent outbursts of up to $10^{-4} M_{\odot} \mathrm{yr}^{-1}$ (Hartmann et al. 1993, pp. 497-518). The bursts are predicted as a consequence of unstable pile-up of gas in the inner disk, which then intermittently releases a cascade of material onto the star due to viscous-thermal disk instabilities (Bell \& Lin 1994; Zhu et al. 2009; D'Angelo \& Spruit 2010). The burst frequency and perhaps amplitude decline over time (e.g., Hartmann \& Kenyon 1996; Vorobyov \& Basu 2015).

While most of the stellar mass is thought to accumulate in the early phases of protostellar evolution, accretion at rates of $10^{-10}-10^{-6} M_{\odot} \mathrm{yr}^{-1}$ persists through the T Tauri phase (ages up to a few megayears), with less frequent bursts (Hartmann et al. 2016). The currently accepted picture of $T$ Tauri star accretion involves magnetic funnel flows channeling gas from the inner disk onto the central star. Where this material impacts the surface, shocks arise, and thermal hot spots form. There may be one spot near each magnetic pole, or multiple spot complexes that are distributed about the stellar surface. The number and geometry of the funnel flows is thought to depend on the accretion rate (e.g., Romanova et al. 2008).

Empirically, time series monitoring of young stellar objects (YSOs; ages $<1-10 \mathrm{Myr}$ ) has an extensive history. It has been

\footnotetext{
${ }^{4}$ Current address: Joint ALMA Observatory, Av. Alonso de Córdova 3107, Vitacura, Santiago, Chile.
}

known since or before, e.g., Joy (1949) that T Tauri stars display flux variations at a wide variety of timescales and magnitudes. The outbursting FU Ori stars and their lower amplitude, repeating cousins the EX Lup stars, are at the extreme end of the variability spectrum-and rare. More common photometric variability is characterized by smaller amplitude and shorter timescale fluctuations. The studies of Herbst et al. (1994), Grankin et al. (2007), Rucinski et al. (2008), and Findeisen et al. (2013) have illustrated and quantified much of the "typical" young star photometric phenomena occurring from sub-hour to multi-decade timescales. One cause of the routine brightness variations is sporadic infall of material from the surrounding disk.

Even in their predominant low-state accretion phases, young stars are understood as variable accretors. The photometric variability is complemented by highly variable emission line profiles and veiling (e.g., Chou et al. 2013; Costigan et al. 2014) including in stars with relatively low accretion rates such as TW Hya and V2129 Oph (Alencar \& Batalha 2002; Alencar et al. 2012).

Nevertheless, the variability timescales and accretion rate changes remain poorly quantified for typical young accreting star/disk systems. There may be a continuum of "burst" behavior with a range of amplitudes and timescales that have not yet been appropriately sampled or appreciated in existing ground-based data sets. While most of the stellar mass has been assumed to accumulate in the episodic and dramatic fashion of the rare large FU Ori and Ex Lup type events, the role and implications of discrete lower amplitude accretion events (e.g., Stauffer et al. 2014) and continuously stochastic accretion behavior (e.g., Stauffer et al. 2016) is not well understood in 
the context of stellar mass accumulation and inner disk evolution.

In probing accretion variability, space-based photometric campaigns have several advantages over ground-based work, including near-continuous sampling (versus interruptions for daytime, weather, etc.), higher measurement precision, and fainter signal detection limits. A detailed analysis of optical and infrared variability among disk-bearing stars in the $\sim 3 \mathrm{Myr}$ NGC 2264 was conducted by Cody et al. (2014) based on a 40day optical time series from the CoRoT space telescope at 10 minute cadence, along with 30 days of Spitzer Space Telescope monitoring at 100 minute cadence. These data enabled an unprecedented view of YSO brightness changes on a variety of timescales. Among the detected variability groups was a new class of "stochastic accretion burst" light curves-dominated by brightening events with durations of $0.1-1$ days and amplitudes of $5 \%-50 \%$ of the quiescent flux value (Stauffer et al. 2014). It was speculated that these events were caused by the unsteady infall of material onto the stellar surface, as predicted by Romanova et al. (2011). They may thus represent "normal" discrete accretion variations and bursts, in contrast to the FU Ori and EX Lup outbursts described above.

The NASA $K 2$ mission Campaign 2 observations included the young $\rho$ Ophiuchus molecular cloud region at $<1-2 \mathrm{Myr}$, and the adjacent Upper Scorpius OB association, which is debated from analysis of HR diagrams to be either 3-5 Myr based on the low-mass stellar population (e.g., Preibisch et al. 2002; Herczeg \& Hillenbrand 2015) or $\sim 11$ Myr based on the solar and super-solar mass population (Pecaut et al. 2012); the latter age is beginning to be favored by results on eclipsing binaries (Kraus et al. 2015; David et al. 2016) and asteroseismology (Ripepi et al. 2015). By sampling stars with ages comparable to and extending to much older than NGC 2264 (in $\rho$ Oph and Upper Sco, respectively), the $K 2$ time series data can be used to compare accretion burst behavior as a function of age and therefore presumably disk properties, which are expected to evolve with time.

We report here on a continuum of accretion burst behavior among members of $\rho$ Oph and Upper Sco. We observe discrete brightening events that range in amplitude from 0.1 to $2.5 \mathrm{mag}$ and in timescale from $<1$ day to $>1$ week. We demonstrate that the burst phenomenon is seen only in those stars with evidence for strongly accreting disks, distinct from the typical disks in the region with weaker infrared excess and $\mathrm{H} \alpha$ emission. Section 2 contains a description of the $K 2$ observations and pixel file processing, and of follow-up high dispersion spectroscopy. Section 3 presents the light-curve analysis and identification of burst type variables, Section 4 presents a discussion of the corresponding accretion and disk properties, and Section 5 presents the spatial and time domain characteristics of bursting sources. We discuss the implications of these observations in Section 6 and summarize the results in Section 7.

\section{Observations}

\subsection{K2 Photometry}

The $K 2$ mission (Howell et al. 2014) observed nearly 2000 stars in the young $\rho$ Ophiuchus and Upper Scorpius regions during Campaign 2. We have mainly considered objects submitted under programs GO2020, GO2047, GO2052, GO2056, GO2063, and GO2085 of the Campaign 2 solicitation, which comprise both secure cluster members and less secure candidates. We later noted aperiodically variable stars among a number of other programs targeting cool dwarfs and therefore added objects from programs GO2104, GO2051, GO2069 GO2029, GO2106, GO2089, GO2092, GO2049, GO2045, GO2107, GO2075, and GO2114 if they also had proper motions consistent with Upper Sco. A. M. Cody (2017, in preparation) cull some of the less confident young star candidates using WISE photometry to eliminate giant stars and other contaminants. This vetting results in a reduced set of $1443 \rho$ Oph and Upper Sco candidate members.

For each star in this set, we downloaded the target pixel file (TPF) from the Mikulski Archive for Space Telescopes (MAST). Each target is stored under its Ecliptic Plane Input Catalog (EPIC) identification number, as listed in Table 1. Data for each object includes $3811 \sim 10 \times 12$ pixel stamp images, obtained between 2014 August 23 and November 10 .

Since the loss of a second reaction wheel during the Kepler mission in May of 2013, telescope pointing for $K 2$ has suffered reduced stability and requires corrective thruster firings approximately every six hours. We find a corresponding target centroid drift at a rate of $\sim 0$ !" 1 , or $\sim 0.02$ pixels, per hour. While this movement is relatively small, associated detector sensitivity variations at the few percent level per pixel compromise the otherwise exquisite photometry, introducings jumps in measured flux on the same six-hour timescales.

It is helpful to track the $x-y$ position drift for each star over time because this can be used for aperture placement and later detrending of the light curves. TPF headers provide a rough world coordinate system solution, which is the same for all images, but these are not precise enough to center the target. We therefore cut out a $5 \times 5$ pixel region around the specified target position, and used this to calculate a flux-weighted centroid.

We carried out photomety with moving apertures, the centers of each specified by the measured centroid locations. This approach helps to minimize the effect of detector drift on the photometry. Circular apertures were used with radii ranging from 1.0 to 4.0 pixels, in intervals of 0.5 pixels. We found that photometric noise levels after detrending for position jump effects were generally minimized with the 2 pixel aperture, though for a few objects we selected the 1.5 or 3 pixel apertures. These sizes have the additional advantage of being small enough so as to avoid flux contamination from other stars lying $\sim 12^{\prime \prime}$ away.

To clean the data, we discarded the first 93 light-curve points, for which the pixel positions were particularly errant compared to the rest of the time series. We also removed points with detector anomaly flags. Finally, we pruned points lying more than five standard deviations off the median light-curve trend. This was accomplished by median smoothing on $\sim 2$ day timescales, removing outliers, and then adding the median trend back in.

In general, our raw moving aperture photometry consists of lower levels of pointing-related systematic jitter than for the fixed aperture case. For all of the stars discussed in this work, the amplitude of intrinsic variability dwarfs these systematics, 
Table 1

Young Stars Exhibiting Bursting Behavior in $K 2$ Campaign 2

\begin{tabular}{|c|c|c|c|c|c|c|c|}
\hline EPIC id & 2MASS id & Other ids & $\mathrm{SpT}$ & $\begin{array}{l}\text { EW H } \alpha \\
(\AA)\end{array}$ & $\begin{array}{l}\mathrm{H} \alpha 10 \% \\
\left(\mathrm{~km} \mathrm{~s}^{-1}\right)\end{array}$ & References & Region \\
\hline 203382255 & $\mathrm{~J} 16144265-2619421$ & & M4-M5.5 & -77 & 154 & 1 & USco \\
\hline 203725791 & $\mathrm{~J} 16012902-2509069$ & USco CTIO 7 & M2/M3.5 & $-\mathbf{1 7 0},-129$ & 437 & 1,2 & USco \\
\hline 203786695 & $\mathrm{~J} 16245974-2456008$ & WSB 18 & M3.5 & $-8.4,-140$ & $\cdots$ & 3 & $\rho \mathrm{Oph}$ \\
\hline 203789507 & J15570490-2455227 & & .. & $\ldots$ & $\ldots$ & & USco \\
\hline 203794605 & J16302339-2454161 & WSB 67 & M3.5-M5 & -69 & 485 & 1 & $\rho \mathrm{Oph}$ \\
\hline 203822485 & J16272297-2448071 & WSB 49, MHO 2111, DROXO 57 & M4.25 & -37 & $\ldots$ & 4 & $\rho \mathrm{Oph}$ \\
\hline 203856109 & J16095198-2440197 & & M5-M5.5 & -15 & 155 & 1 & USco \\
\hline 203899786 & J16252434-2429442 & V852 Oph, SR 22, DoAr 19, WSB 23 & $\mathrm{M} 4.5 / \mathrm{M} 3$ & $-31,-170$ & $\cdots$ & $4,5,6$ & $\rho$ Oph \\
\hline 203905576 & J16261886-2428196 & VSSG 1, Elias 20, YLW 31, ISO-Oph 24, & $\begin{array}{r}\text { K7-mid- } \\
\text { M/M0 }\end{array}$ & -70 & 416 & 1,7 & $\rho \mathrm{Oph}$ \\
\hline 203905625 & $\mathrm{~J} 16284527-2428190$ & $\begin{array}{l}\text { IRAS } 16233-2421, \text { MHO } 2103 \\
\text { V853 Oph, SR 13, DoAr 40, WSB 62, } \\
\text { ISO-Oph 199, HBC } 266\end{array}$ & M3.75 & $-30,-48,-46$ & $\cdots$ & 4 & $\rho \mathrm{Oph}$ \\
\hline 203913804 & $\mathrm{~J} 16275558-2426179$ & $\begin{array}{l}\text { V2059 Oph, DoAr 37, SR 10, ISO-Oph } 187, \\
\text { WSB 57, YLW 56, HBC } 265, \text { SVS } 1771\end{array}$ & M2 & $-43,-56,-108$ & $\cdots$ & 4,8 & $\rho$ Oph \\
\hline 203928175 & $\mathrm{~J} 16282333-2422405$ & SR $20 \mathrm{~W}$ & K5 & -35 & $\ldots$ & 3 & $\rho$ Oph \\
\hline 203935537 & $\mathrm{~J} 16255615-2420481$ & $\begin{array}{l}\text { V2058 Oph, DoAr 20, Elias 13, } \\
\text { SR 4, WSB 25, YLW 25, IRAS 16229-2413, } \\
\text { MHA 365-12, ISO-Oph } 6\end{array}$ & K4.5 & $-220,-87,-67$ & $\ldots$ & 4 & $\rho \mathrm{Oph}$ \\
\hline 203954898 & $\mathrm{~J} 16263682-2415518$ & ISO-Oph 51 & M0 & -10 & $\ldots$ & 9 & $\rho \mathrm{Oph}$ \\
\hline 204130613 & J16145026-2332397 & BV Sco & M4.5 & -108 & $\ldots$ & 10 & USco \\
\hline 204226548 & J15582981-2310077 & USco CTIO 33, USco 42 & M3 & $-158,-250$ & $\cdots$ & 11,12 & USco \\
\hline 204233955 & $\mathrm{~J} 16072955-2308221$ & & M3 & -150 & $\cdots$ & 10 & USco \\
\hline 204342099 & $\mathrm{~J} 16153456-2242421$ & VV Sco, IRAS 16126-2235, PDS 82a & M1/K9-M0 & $-20,-31$ & 337 & 13,1 & USco \\
\hline 204347422 & J16195140-2241266 & & $\cdots$ & $\cdots$ & $\cdots$ & & USco \\
\hline 204360807 & $\mathrm{~J} 16215741-2238180$ & & M6 & -140 & 341 & 1 & USco \\
\hline 204397408 & J16081081-2229428 & & M5.75/M5 & $-22,-49,-31$ & $\cdots$ & $10,14,15$ & USco \\
\hline 204440603 & $\mathrm{~J} 16142312-2219338$ & & M5.75 & -95 & $\ldots$ & 10 & USco \\
\hline 204830786 & J16075796-2040087 & IRAS $16050-2032$ & M1/G6-K5 & $-357,-\mathbf{1 6 5}$ & 684 & 16,1 & USco \\
\hline 204906020 & J16070211-2019387 & KSA 68 & M5 & $-8,-30$ & $\cdots$ & 11,17 & USco \\
\hline 204908189 & J16111330-2019029 & & M1/M3 & -160 & 324 & 1,18 & USco \\
\hline 205008727 & J16193570-1950426 & & K7-M3 & -55 & 322 & 1 & USco \\
\hline 205061092 & $\mathrm{~J} 16145178-1935402$ & & M5-M6 & -70 & 173 & 1 & USco \\
\hline 205088645 & J16111237-1927374 & & M5, M6 & $-50,-50$ & $\ldots$ & 12,19 & USco \\
\hline 205156547 & J16121242-1907191 & & M5-M6 & -15 & 127 & 1 & USco \\
\hline
\end{tabular}

Note. Stars in the $K 2$ Campaign 2 burster sample, in order of EPIC id. EPIC 203786695/2MASS J16245974-2456008 has a companion at 1!" 1 separation, and the two $\mathrm{H} \alpha$ values belong to the distinct components of the system. The $\mathrm{H} \alpha 10 \%$ widths in column 6 are derived from data presented in this paper. We have highlighted in bold the other new values derived as part of this work.

References. (1) This work, (2) Rizzuto et al. (2015), (3) Brandner \& Zinnecker (1997), (4) Wilking et al. (2005), (5) Prato (2007), (6) Martin et al. (1998), (7) Andrews et al. (2010), (8) Appenzeller et al. (1983), (9) Erickson et al. (2011), (10) Lodieu et al. (2011), (11) Dahm \& Carpenter (2009), (12) Preibisch et al. (2002), (13) Preibisch et al. (1998), (14) Slesnick et al. (2008), (15) Dahm et al. (2012), (16) Kraus \& Hillenbrand (2009), (17) Preibisch et al. (2001), (18) Luhman \& Mamajek (2012), (19) Martín et al. (2010).

and no further corrections are needed. However, this is not the case for the less variable cluster members, which form a control population for comparison of variability demographics. In these light curves, a prominent sawtooth pattern appears on the $\sim 6 \mathrm{hr}$ timescales corresponding to thruster firings, an effect that was mitigated with the detrending procedure described in Aigrain et al. (2016), as described in A. M. Cody (2017, in preparation).

In one exceptional case (EPIC 203954898/2MASS J1626 3682-2415518), the light of a highly variable star was contaminated by a close neighbor $8^{\prime \prime}$ away. Single pixel photometry shows that this object undergoes high-amplitude bursts, while the neighboring star is relatively constant and slightly fainter. Restricting the aperture to encompass only EPIC 203954898 results in degraded precision. We therefore used a 3 pixel aperture encircling both stars, and then removed the average flux of the companion by scaling the data to match the amplitude of the bursts in the single pixel light curves (the lower precision here affects only the detailed light-curve morphology and not its overall amplitude). The result is a light curve with maximum burst amplitude of nearly eight times the quiescent flux level.

\subsection{New Spectra and Compiled Spectroscopic Data}

We collected both archival and new spectroscopic data at high dispersion with the Keck/HIRES spectrograph (Vogt et al. 1994). The new observations were obtained on 2016 May 17 and 20, 2015 June 1 and 2, or 2013 June 4, UT, and covered the spectral range of $\sim 4800 \AA$ to $9200 \AA$ at 
a resolution of $R \approx 36,000$. The images were processed and the spectra were extracted and calibrated using the makee software written by Tom Barlow. $\mathrm{H} \alpha$ emission line strengths and spectral type estimates are tabulated in Table 1; other emission lines that were observed in these high dispersion data are discussed in the Appendix notes on individual stars. Table 1 as well as the notes section includes literature information in addition to our spectroscopic findings.

\subsection{Speckle Imaging}

We obtained high-resolution speckle imaging for six of our young stars to assess multiplicity properties. Our observations used the Differential Speckle Survey Instrument (DSSI; Horch et al. 2011) on the Gemini-South telescope in 2016 June. Speckle observations were simultaneously made in two medium band filters with central wavelengths and bandpass FWHM values of $\left(\lambda_{c}, \delta \lambda\right)=(692,47)$ and $(883,54) \mathrm{nm}$. Each star was observed for approximately 10 minutes during which time we obtained three to five image sets consisting of 1000, $60 \mathrm{~ms}$ simultaneous frames. These observations were made during clear weather at airmass 1.0-1.3, when the native seeing was $0.4-0.6$ arcsec. Details of speckle observations using the Gemini telescope and our data reduction procedures can be found in Horch et al. (2012) and Howell et al. (2011).

\section{Identification of Accretion Bursts}

We conducted visual examinations of all 1443 young star light curves in order to identify stars in a "bursting" state. Such objects were selected by identifying behavior consistent with that presented in Cody et al. (2014) and Stauffer et al. (2014). To confirm our visually identified bursters, we also computed quantitative variability metrics, specifically, the $M$ and $Q$ statistics defined by us in the papers above. $M$ describes the degree of symmetry of the light curve about its mean value. It is calculated by determining the ratio of the mean of magnitude data in the top and bottom deciles to the median of all lightcurve points. $M$ achieves negative values when there is a significant number of points brighter than the median, but not so many faint points. Unlike what was done in Cody et al. (2014), we calculated the $M$ statistic from flux, rather than magnitude values. The main effect of this change is to lower $M$ values (by $\sim 10 \%$ on average), since the magnitude to flux conversion makes bright peaks more pronounced. We argue that flux units are a more natural choice here, as flux correlates with luminosity and accretion rate. Bursting light curves are highly asymmetric with frequent flux increases over the mean, resulting in $M$ values from approximately -0.3 to -1.3 . While the boundary is somewhat subjective, all objects selected byeye meet the previously defined $M<-0.25$ criterion for burster status.

The $Q$ statistic defined in Cody et al. (2014) describes tendency toward or away from periodicity over the time series. It is a measurement of how much the standard deviation shrinks when the light curve is phased to its dominant periodicity and the associated pattern is repeated and subtracted out from the raw time series. Strictly periodic behavior (i.e., complete removal of the phase pattern) returns $Q=0$, while light curves with no repeating behavior have $Q=1$. In a few cases for which the light curves are entirely aperiodic, this removal process actually increases the underlying standard deviation; this is why the computed $Q$ value is occasionally greater than 1.0. In previous work, we have denoted light curves with moderate $Q$ values of $0.15-0.60$ as "quasi-periodic." We emphasize here that this range is somewhat subjective and was based on a by-eye analysis of CoRoT data on the NGC 2264 cluster. As explained in A. M. Cody et al. (2017, in preparation), the present $K 2$ data set contains some objects with $Q>0.6$ that nevertheless display repeating components upon visual examination.

The $M$ and $Q$ values for the selected bursters are provided in Table 2. They are also plotted in Figure 1, alongside the values for other disk-bearing young stars in the $K 2$ field, as selected in A. M. Cody (2017, in preparation). The $K 2$ burster sample behavior ranges from periodic to quasi-periodic to aperiodic, with light curves tending mostly toward aperiodicity. Objects falling in the bursting section of the diagram but not highlighted as such tend to be long-timescale variables for which the trend removal failed. We favor our by-eye classification over the $M$ and $Q$ statistics here and thus do not consider these bursters. Several other objects with highly negative $M$ values are dominated by periodic modulation and only display zero or one bursting event. We also leave these out of the sample, in light of classification ambiguity. Overall, 18 objects with $M<-0.25$ (i.e., a value that would qualify them as busters) were removed from the sample. Most of these may be seen in Figure 1 as the black points lying above the $M=-0.25$ line (apart from five that are hidden under orange burster points); the majority are only marginally above it.

In total, we have selected 29 stars as bursters in $\rho$ Oph and Upper Sco; division into the two regions was based on a $1.2^{\circ} \times 1.2^{\circ}$ square surrounding the position R.A. $=246.79$, decl. $=-24.60$ to define the extent of $\rho$ Oph (see A. M. Cody 2017 , in preparation). We list the basic properties of the bursters in Table 1 and show their light curves in Figure 2. The burster class exhibits several subsets of behavior, with some light curves displaying a nearly continuous series of events, and others exhibiting more discrete brightening events. We discuss the timescales of bursting in Section 6.

\section{Disk and Accretion Properties of the Burst Sample}

A hypothesis for the short-lived, often repeatable, brightening events in our $29 K 2$ light curves is that they are caused by episodic accretion from the circumstellar disk onto the young star. A specific requirement for the accretion-driven burst hypothesis is that the objects exhibit both infrared excess indicative of circumstellar dust, serving as the reservoir for the accretion, and either ultraviolet excess or line emission from hot gas in the nearby circumstellar environment. The dust criterion is satisfied by our burster sample, as all stars have been already selected as infrared excess sources in A. M. Cody (2017, in preparation). As shown in that work, there are 344 disk-bearing stars in the entire $K 2$ Campaign 2 sample, of which 299 are bright enough to obtain light curves. This number includes all 29 burster stars identified here. Therefore, the fraction of bursters among the total disk-bearing sample is at least $8 \pm 2 \%$. The error comes from consideration of the Poisson uncertainties. These values can also be considered separately for the $\rho$ Oph (128 disked stars; 92 with light curves) and Upper Sco (216 disked stars; 207 with light curves) 
Table 2

Light-curve Metrics for Bursting Young Stars in K2 Campaign 2

\begin{tabular}{|c|c|c|c|c|c|c|c|c|}
\hline EPIC id & 2MASS id & $\begin{array}{c}\text { Amplitude } \\
\text { (Norm. Flux) }\end{array}$ & $Q$ & $M$ & $\begin{array}{l}\text { Timescale } \\
\text { (day) }\end{array}$ & $\begin{array}{l}\text { Duration } \\
\text { (day) }\end{array}$ & Duty Cycle & $\begin{array}{c}\text { Period } \\
\text { (day) }\end{array}$ \\
\hline 203382255 & J16144265-2619421 & 1.43 & 1.00 & -1.14 & $>77.74$ & 7.95 & 0.08 & $\ldots$ \\
\hline 203725791 & $\mathrm{~J} 16012902-2509069$ & 0.68 & 0.87 & -0.29 & 5.18 & 1.90 & 0.66 & $\ldots$ \\
\hline 203786695 & J16245974-2456008 & 0.25 & 1.00 & -0.59 & 5.52 & 4.54 & 0.43 & $\cdots$ \\
\hline 203789507 & J15570490-2455227 & 0.36 & 0.97 & -0.41 & 9.12 & 4.45 & 0.06 & $\ldots$ \\
\hline 203794605 & J16302339-2454161 & 0.87 & 0.55 & -0.25 & 4.64 & 3.67 & 0.93 & 4.46 \\
\hline 203822485 & J16272297-2448071 & 0.62 & 0.84 & -0.29 & 9.36 & 7.66 & 0.90 & $\cdots$ \\
\hline 203856109 & J16095198-2440197 & 0.35 & 1.00 & -1.00 & 3.11 & 5.25 & 0.15 & $\cdots$ \\
\hline 203899786 & J16252434-2429442 & 1.13 & 0.61 & -0.83 & 6.42 & 3.98 & 0.96 & 5.95 \\
\hline 203905576 & J16261886-2428196 & 3.85 & 1.00 & -0.66 & 7.48 & 5.97 & 0.88 & $\ldots$ \\
\hline 203905625 & $\mathrm{~J} 16284527-2428190$ & 0.32 & 1.0 & -0.31 & 7.80 & 5.17 & 0.72 & $\ldots$ \\
\hline 203913804 & $\mathrm{~J} 16275558-2426179$ & 0.41 & 1.0 & -0.37 & 5.47 & 3.56 & 0.70 & $\ldots$ \\
\hline 203928175 & $\mathrm{~J} 16282333-2422405$ & 3.19 & 0.54 & -0.66 & 4.68 & 2.53 & 0.73 & 4.39 \\
\hline 203935537 & $\mathrm{~J} 16255615-2420481$ & 0.24 & 1.00 & -0.31 & 4.92 & 3.09 & 0.69 & $\ldots$ \\
\hline 203954898 & $\mathrm{~J} 16263682-2415518$ & 6.82 & 0.61 & -1.35 & 31.17 & 6.84 & 0.43 & 20.83 \\
\hline 204130613 & J16145026-2332397 & 1.87 & 0.85 & -0.35 & 3.43 & 0.90 & 0.62 & $\cdots$ \\
\hline 204226548 & J15582981-2310077 & 0.47 & 1.00 & -0.53 & 8.54 & 3.44 & 0.73 & $\ldots$ \\
\hline 204233955 & $\mathrm{~J} 16072955-2308221$ & 1.99 & 0.85 & -0.82 & 3.42 & 2.08 & 0.98 & $\cdots$ \\
\hline 204342099 & J16153456-2242421 & 0.83 & 0.91 & -0.72 & 35.53 & 11.26 & 0.88 & $\cdots$ \\
\hline 204347422 & J16195143-2241332 & 1.38 & 0.75 & -1.11 & 7.23 & 1.65 & 0.10 & 6.94 \\
\hline 204360807 & $\mathrm{~J} 16215741-2238180$ & 0.55 & 0.87 & -0.49 & 5.21 & 2.53 & 0.65 & $\cdots$ \\
\hline 204397408 & J16081081-2229428 & 0.15 & 0.59 & -0.68 & 5.01 & 5.30 & 0.22 & 1.65 \\
\hline 204440603 & $\mathrm{~J} 16142312-2219338$ & 0.43 & 1.00 & -0.93 & 4.55 & 3.15 & 0.28 & $\ldots$ \\
\hline 204830786 & J16075796-2040087 & 1.91 & 1.00 & -0.67 & 14.70 & 4.31 & 0.91 & $\cdots$ \\
\hline 204906020 & J16070211-2019387 & 0.34 & 0.93 & -0.47 & 3.90 & 0.91 & 0.63 & $\cdots$ \\
\hline 204908189 & J16111330-2019029 & 1.28 & 0.76 & -0.59 & 7.99 & 7.97 & 0.90 & 19.23 \\
\hline 205008727 & J16193570-1950426 & 0.91 & 1.00 & -0.68 & 10.25 & 7.93 & 0.76 & $\cdots$ \\
\hline 205061092 & J16145178-1935402 & 0.35 & 1.00 & -0.51 & 4.85 & 1.94 & 0.35 & $\cdots$ \\
\hline 205088645 & J16111237-1927374 & 0.10 & 1.00 & -0.53 & 11.30 & 3.18 & 0.04 & $\cdots$ \\
\hline 205156547 & J16121242-1907191 & 0.16 & 1.00 & -1.01 & 4.38 & 3.62 & 0.23 & $\cdots$ \\
\hline
\end{tabular}

Note. We tabulate basic statistical properties of the burster light curves. $Q$ and $M$ are discussed in Section 3 as well as Cody et al. (2014). Amplitudes represent peakto-peak measurements.

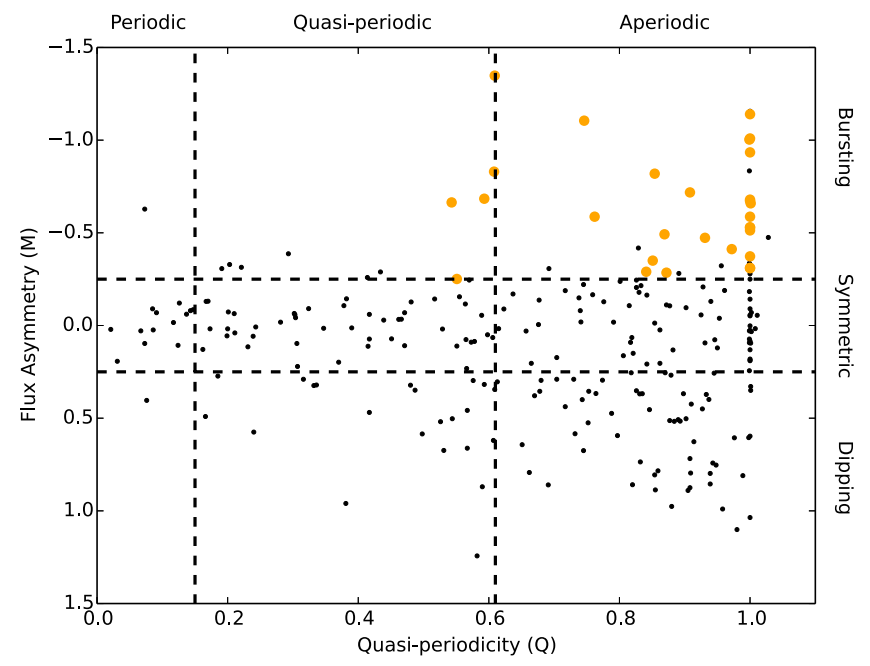

Figure 1. $Q$ and $M$ values for all stars in $\rho$ Oph and Upper Sco observed by $K 2$. Black dashed lines indicate the approximate borders between different variability behaviors, as identified by Cody et al. (2014). Objects identified in this paper as bursters are highlighted in orange; all lie above the $M=0.25$ boundary.

samples. There are 10 bursters in $\rho$ Oph and 19 bursters in Upper Sco; both numbers lead to roughly the same value of $8 \%-10 \%$, with an $\sim 2 \%$ error on this fraction.

\subsection{Circumstellar Dust}

Infrared color-magnitude diagrams can also shed light on what photometric aspects, if any, separate burster stars from other disk-bearing sources. We show the color-color diagrams $J-K$ versus $K-W 3$ and $J-K$ versus $K-W 4$ in Figure 3 and the spectral energy distributions in Appendix Figure 14, to illustrate the strength of emission at farther, cooler locations in the disk. The vast majority of the bursters have excesses in the three longest wavelength WISE bands $W 2, W 3$, and $W 4$ (4.6 $\mu \mathrm{m}, 12 \mu \mathrm{m}$, and $22 \mu \mathrm{m}$, respectively). The individual spectral energy distributions in the Appendix (Figure 14) provide a finer look at the circumstellar flux patterns. This finding is in stark contrast to the overall disk sample, in which only $50 \%$ of objects (or a total of 172) have excesses in all three bands. Thus, the presence of a full, minimally evolved disk appears to be preferred for bursting behavior.

It has traditionally been thought that young stars with prominent fading events are surrounded by nearly edge-on disks; the dust clumps routinely obscure the central star (e.g., Bouvier et al. 2003, 2013), causing decrements in the light curve. Conversely, one might imagine that for disk systems farther from edge-on orientation, we would have a more direct view of the accretion columns and shocks near the stellar poles. This could allow a relatively unfettered observation of accretion bursts. Looking at the selection of 172 full disks 

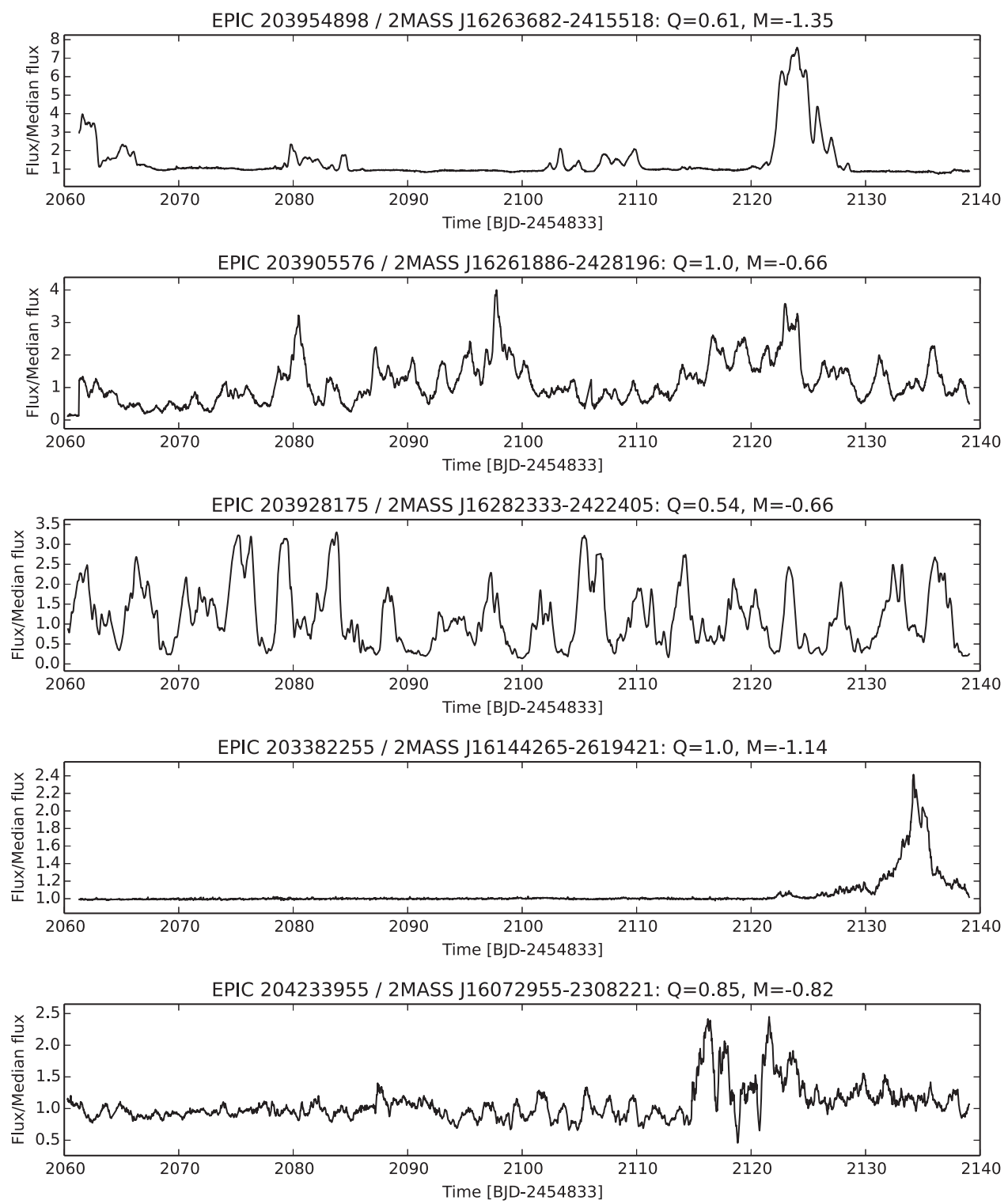

Figure 2. Light curves of selected bursters over the 80-day duration of $K 2$ Campaign 2, in approximate order of amplitude.

identified in A. M. Cody (2017, in preparation), $90 \%$ are variable, but only $17 \%$ are bursting. If this is a reflection of geometric selection, then one might hypothesize that burster disks are viewed at angles ranging from face-on to $\sim 34^{\circ}$. However, this assumes that face-on is the best angle at which to view bursting; as suggested by the ALMA data described below, this may not be the case.

The idea that bursting behavior may be a function of viewing angle can be further explored by considering resolved disk imaging. Five of the bursters discussed in this paper have been observed with ALMA at $0.88 \mathrm{~mm}$ (Carpenter et al. 2014; Barenfeld et al. 2016). EPIC 204830786 (2MASS J160757962040087) has a broad CO $J=3-2$ line detection, with velocities from -17 to $17 \mathrm{~km} \mathrm{~s}^{-1}$; the profile suggests that the disk is not face-on. EPIC 204342099 (2MASS J161534562242421) is the only other disk with a CO detection, albeit a weak one. The broad velocity distribution again suggests that this system is not oriented face-on. The other three disk-bearing sources in our sample (EPIC 204906020/2MASS J16070 211-2019387, EPIC 204226548/2MASS J15582981-2310077,
EPIC 204908189/2MASS J16111330-2019029) have no CO detection. The latter two do show continuum, so CO may be highly depleted or the disks in these cases could be physically small.

The continuum fluxes can also be used to infer disk properties. S. A. Barenfeld (2017, in preparation) infers from modeling SEDs and size constraints that EPIC 204830786 (2MASS J16075796-2040087) has an inclination of $42^{\circ+12}$, while EPIC 204342099 (2MASS J16153456-2242421) is inclined at $43^{\circ}{ }_{-16}$. Dust masses were inferred by Barenfeld et al. (2016) and range from $<0.5 M_{\oplus}$ (EPIC 204906020/ 2MASS J16070211-2019387; non-detection) to $9.3 M_{\oplus}$ in the case of EPIC 204830786/2MASS J16075796-2040087. All five bursters in the ALMA sample are noted as having been classified as full disks (as opposed to evolved or transitional) by Luhman \& Mamajek (2012).

These intermediate inclination values are consistent with the idea proposed above that we are not looking through disk material, which would be expected to produce "dipping" rather than "bursting" light curves. However, they are inconclusive 

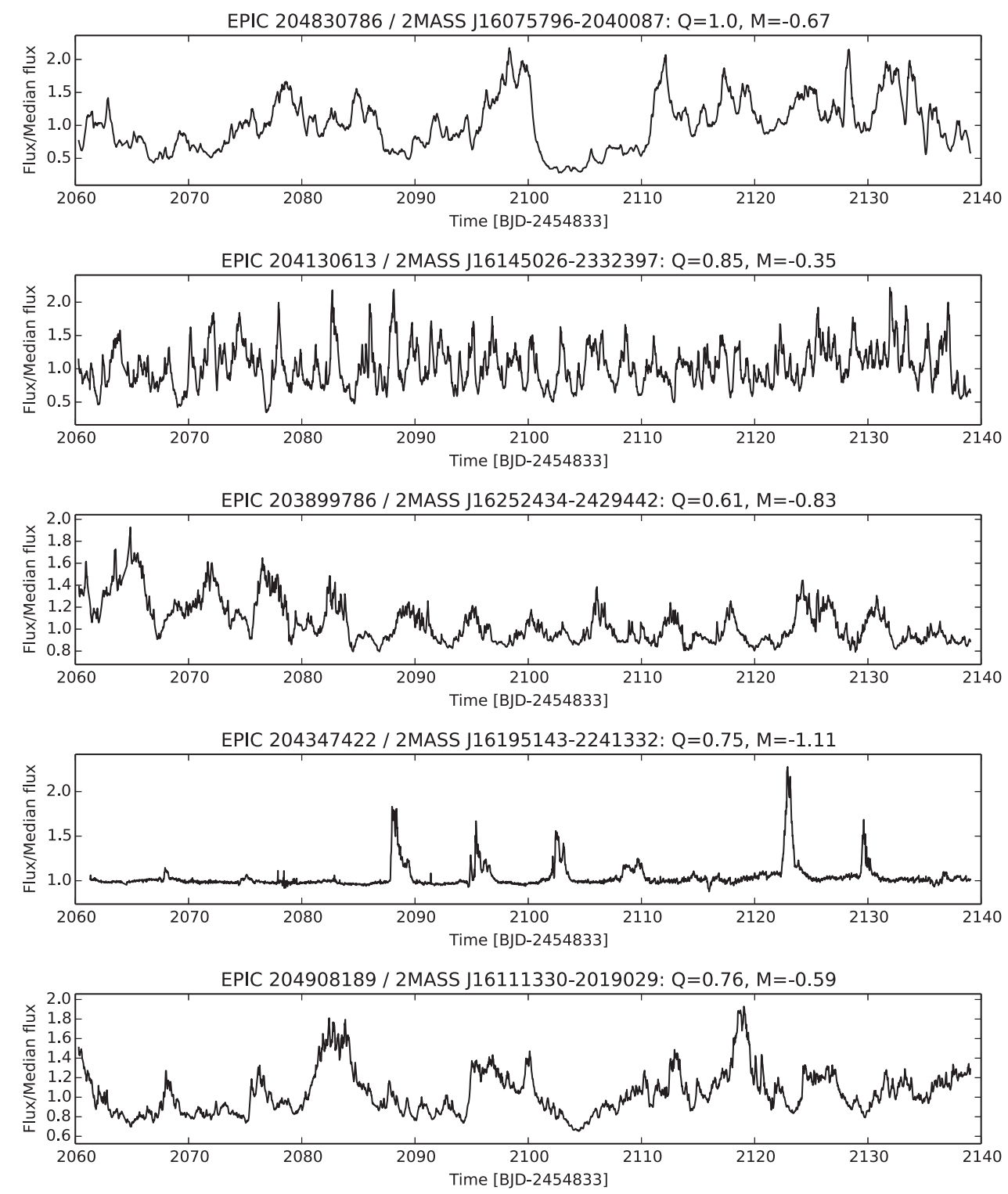

Figure 2. (Continued.)

regarding whether we have a direct view of material accreting onto the central star.

\subsection{Gas and Accretion}

In addition to infrared disk indicators, we obtained spectroscopic data from both the literature and our own highresolution follow-up spectroscopy (Section 2.2). The $\mathrm{H} \alpha$ emission equivalent width (EW) and $10 \%$ width values (Table 1) are indicative of significant accretion. As a control sample, we gathered $\mathrm{H} \alpha \mathrm{EWs}$ for other disk-bearing (but not necessarily bursting) stars in Upper Sco from Rizzuto et al. (2015), Dahm \& Carpenter (2009), and Preibisch et al. (2002). We cross-matched them against our Upper Sco/ $\rho$ Oph $K 2$ WISE excess star list and eliminated any objects not in common. We then compared the $\mathrm{H} \alpha$ values of this general Upper Sco sample with those of the bursters in Figure 4. Since some of the bursters have multiple $\mathrm{H} \alpha$ measurements (see Table 1), we have taken the average of all available values. We find that the bursters occupy a large range of $\mathrm{H} \alpha \mathrm{EW}$ values, from $-10 \AA$ (presumably a low-state value) to several hundred angstroms in emission. The non-burster stars, on the other hand, display weak $\mathrm{H} \alpha$, with EWs primarily from 0 to $-15 \AA$ and a tail out to $-50 \AA$ with one value around $-120 \AA$.

Figure 5 illustrates the emission line profiles for 12 out of the 29 bursters for which we have high-resolution spectra in $\mathrm{H} \alpha$, Ca II $8542 \AA$, and He I $5876 \AA$. White \& Basri (2003) advocated the designation of accreting stars based on $\mathrm{H} \alpha$ line widths at $10 \%$ of the maximum line strength that are larger than $270 \mathrm{~km} \mathrm{~s}^{-1}$. Although all 12 sources have velocities larger than $100 \mathrm{~km} \mathrm{~s}^{-1}$ (see Table 1), only slightly more than onehalf meet the White \& Basri (2003) requirement. There is a tendency for the narrower velocity stars (EPIC 203856109/ 2MASS J16095198-2440197, EPIC 205156547/2MASS 1612 1242-1907191, EPIC 203382255/2MASS J16144265-26194 21, EPIC 205061092/2MASS J16145178-1935402, and EPIC 205008727/2MASS J16193570-1950426) to have lower duty cycles in their burst patterns (Figure 2; where available, $\mathrm{H} \alpha$ velocity and duty cycle are correlated at a significance level of 

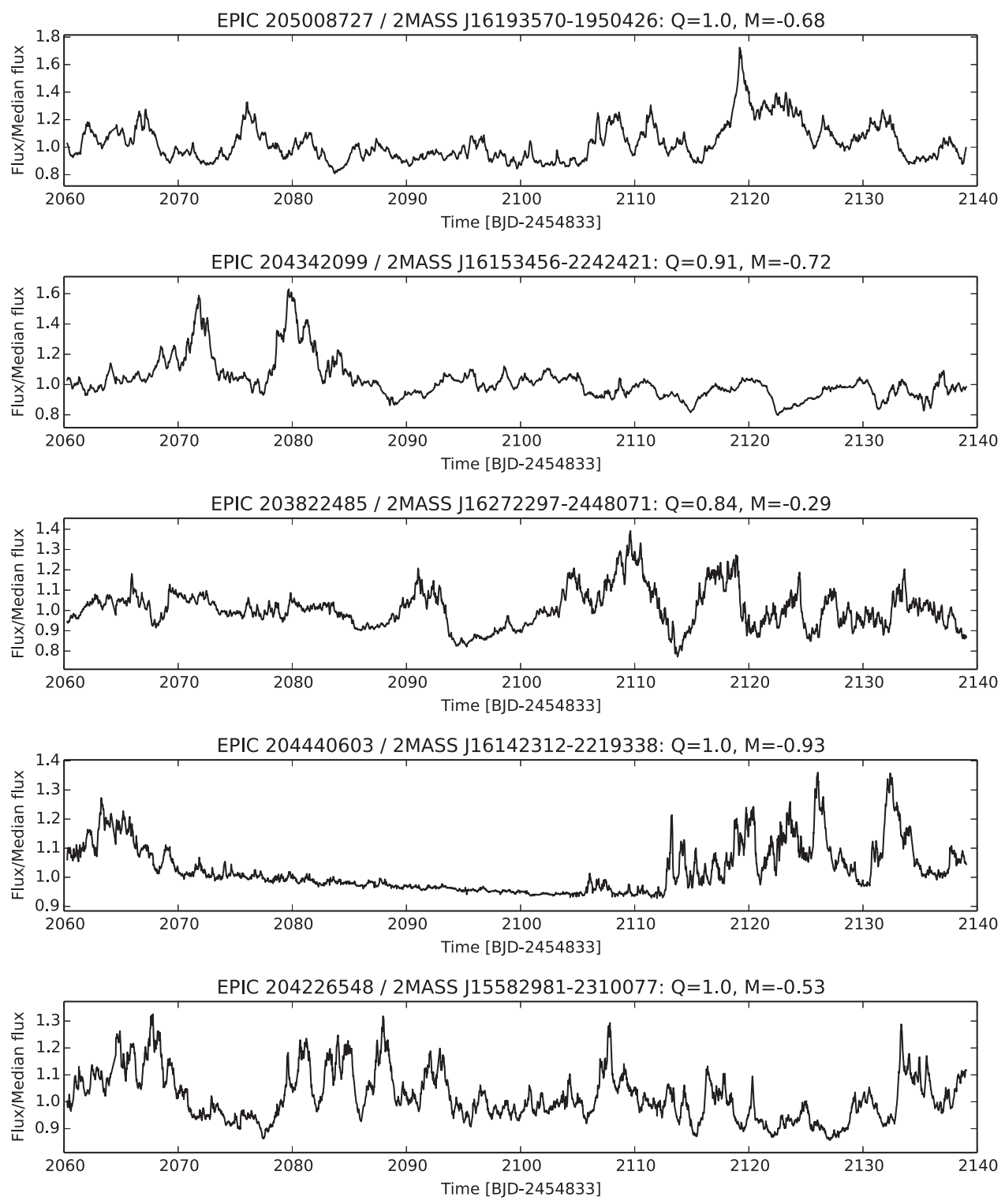

Figure 2. (Continued.)

$\left.1.2 \times 10^{-3}\right)$. It may be that our spectra were taken at non-burst epochs. However, this is speculative at best.

The weak $\mathrm{H} \alpha$ sources all exhibit only narrow component emission in their Ca II profiles, as does EPIC 204342099 (2MASS J16153456-2242421) which has a broad $\mathrm{H} \alpha$ profile. Generally, the broad $\mathrm{H} \alpha$ sources exhibit both broad component and narrow component Ca II. Azevedo et al. (2006) review the classic literature on $\mathrm{Ca}$ II profile morphology in young stars, and discuss magnetospheric models of it. However, our profiles do not seem to exhibit the asymmetries predicted by these models (e.g., blueshifted peaks, redshifted depressions). Notably, our broad-lined Ca II sources also exhibit evidence for forbidden line emission, e.g., [O I] $6300 \AA$.

The morphology of He I emission lines in young stars was studied by Beristain et al. (2001) who also designated narrow line, broad line, and narrow + broad profile categories. Our stars are dominated by their narrow component emission, with widths ranging between 40 and $70 \mathrm{~km} \mathrm{~s}^{-1}$, but some may also have weak broad components that would require line decomposition to characterize. Most of the He I profiles appear to have slight asymmetries, however, in the sense of broader redshifted emission than blueshifted emission with the line peaks at zero-velocity.

Beyond emission line morphology, accretion rates are estimated for a handful of our burster stars by Natta et al. (2006). Values range from $10^{-9.9}$ to $10^{-6.7} M_{\odot} \mathrm{yr}^{-1}-\mathrm{a}$ large range. It should be noted that neither the $\mathrm{H} \alpha$ EWs presented above nor the accretion rates referenced here were necessarily measured during a time when the stars were undergoing bursting events. Thus it is plausible that accretion is preferentially high in these sources, but only at certain times.

Under the hypothesis that burst events in light curves are due to higher than average mass flow, we can convert the flux to a quantitative increase in the mass accretion rate. This requires several assumptions. First, we assign to all stars in our sample a low-level baseline accretion rate, $\dot{M}_{\text {low }}$, which then increases to a larger rate $\dot{M}_{\text {high }}$ during bursts. We assume that this increase 
EPIC 203856109 / 2MASS J16095198-2440197: Q=1.0, M=-1.0

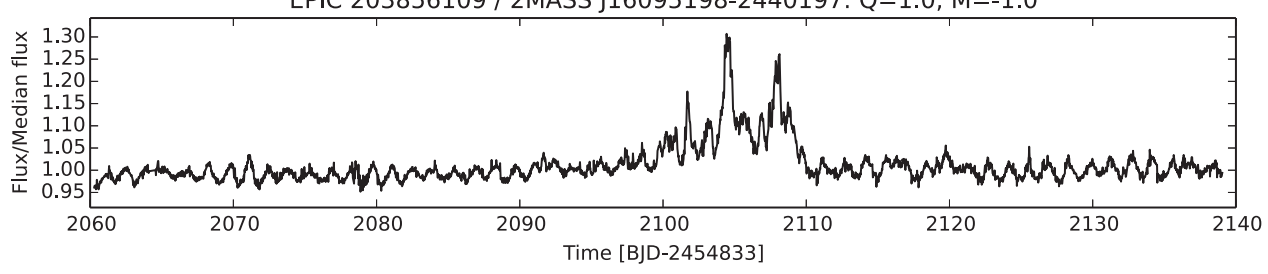

EPIC 203789507 / 2MASS J15570490-2455227: Q=0.97, M=-0.41

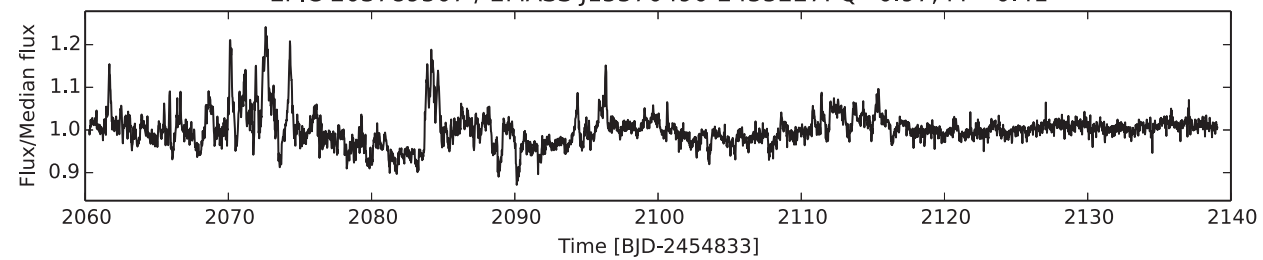

EPIC 203725791 / 2MASS J16012902-2509069: Q=0.87, $M=-0.29$

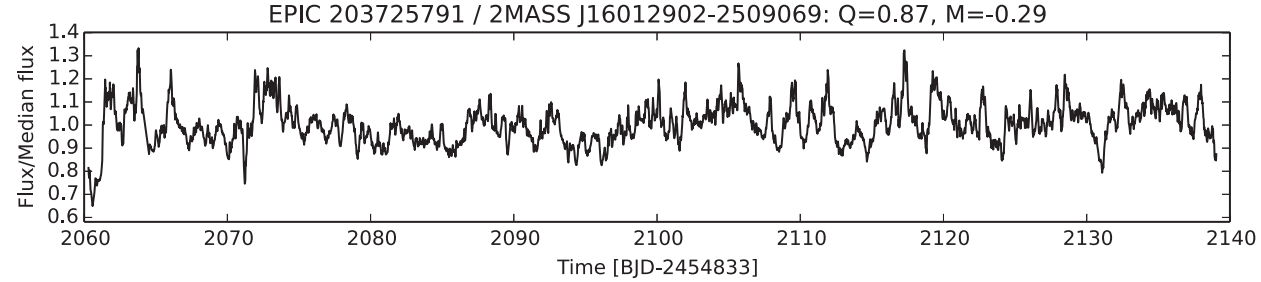

EPIC 204360807 / 2MASS J16215741-2238180: $Q=0.87, M=-0.49$

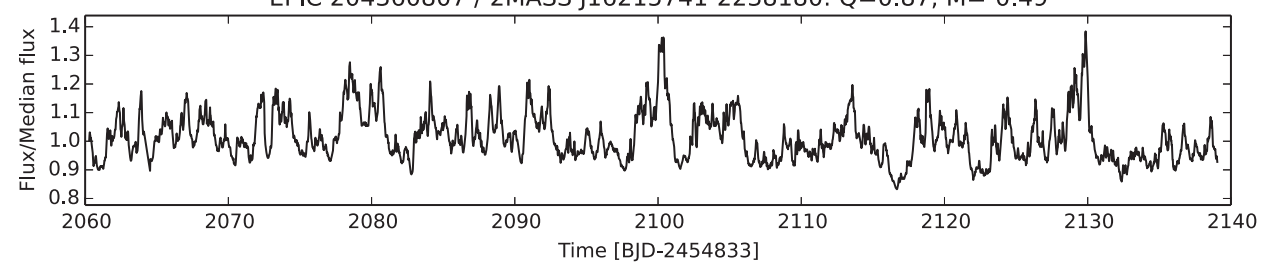

EPIC 205088645 / 2MASS J16111237-1927374: Q=1.0, $M=-0.53$

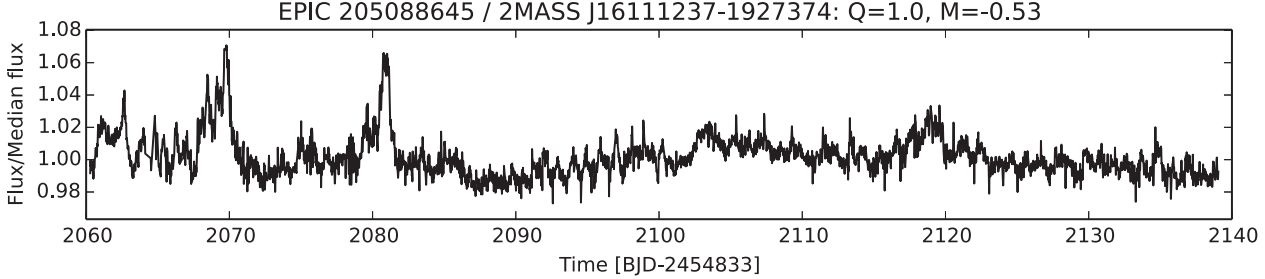

Figure 2. (Continued.)

in mass flow can be equated to the ratio of the accretion flux $F_{\text {acc }}$ in and out of the burst state through the accretion luminosity, $L_{\text {acc}}$ :

$$
\frac{\dot{M}_{\text {high }}}{\dot{M}_{\text {low }}}=\frac{L_{\text {acc,high }}}{L_{\text {acc,low }}} \sim \frac{F_{\text {acc,high }}}{F_{\text {acc,low }}} .
$$

The accretion luminosity is related to the stellar mass $M_{*}$ and radius $R_{*}$ by $L_{\text {acc }}=1.25\left(G M_{*} \dot{M} / R_{*}\right)$, where the pre-factor is that appropriate for an assumed magnetospheric accretion scenario.

The measured flux density $F$ (i.e., in the Kepler band) contains contributions from both accretion and the underlying stellar luminosity, $L_{*}$. We label the measured flux ratio " $r$ ":

$$
r \equiv \frac{F_{\text {high }}}{F_{\text {low }}}=\frac{F_{*}+F_{\text {acc,high }}}{F_{*}+F_{\text {acc,low }}} .
$$

$F_{\text {low }}$ and $F_{\text {high }}$ are approximately the minimum and maximum flux values, respectively, attained in a given light curve. To determine $F_{\text {acc,high }} / F_{\text {acc,low }}$ and hence $\dot{M}_{\text {high }} / \dot{M}_{\text {low }}$, we must estimate the ratio of stellar to accretion luminosity. This quantity has been studied by, e.g., Natta et al. (2014) and we adopt the fit based on their Figure 2. For each star in the burster sample, we estimate the stellar luminosity by considering the $J$-band magnitudes of similar spectral type non-accreting young stars in the $K 2$ Campaign 2 set, and applying bolometric and extinction corrections as outlined in Natta et al. (2006). Combining this with Equations (1) and (2), it can be shown that

$$
\frac{\dot{M}_{\mathrm{high}}}{\dot{M}_{\mathrm{low}}} \sim \frac{L_{*}}{L_{\mathrm{acc}}}(r-1)+r .
$$

Given our estimates of $L_{*} / L_{\text {acc }}$ and $r$ as measured from the $K 2$ photometry, we have calculated the increase in mass accretion rate during bursts for each of our sources. Figure 6 illustrates the resulting values as a function of spectral type, in cases where this is known to a subclass or better. We find that 
EPIC 203794605 / 2MASS J16302339-2454161: Q=0.55, M=-0.2

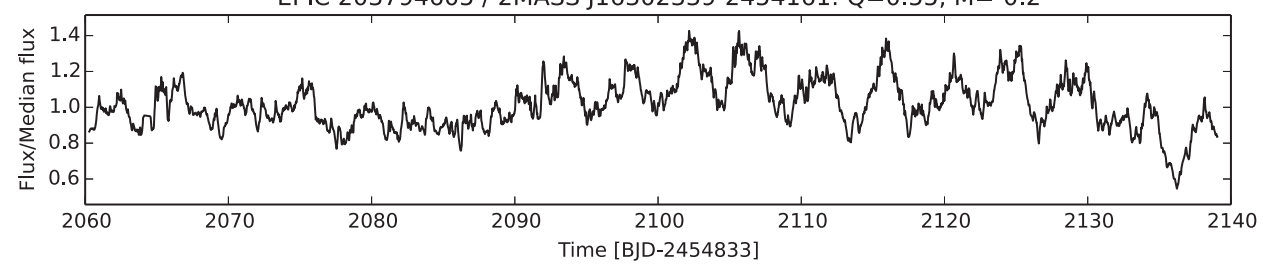

EPIC 205061092 / 2MASS J16145178-1935402: Q=1.0, $M=-0.51$

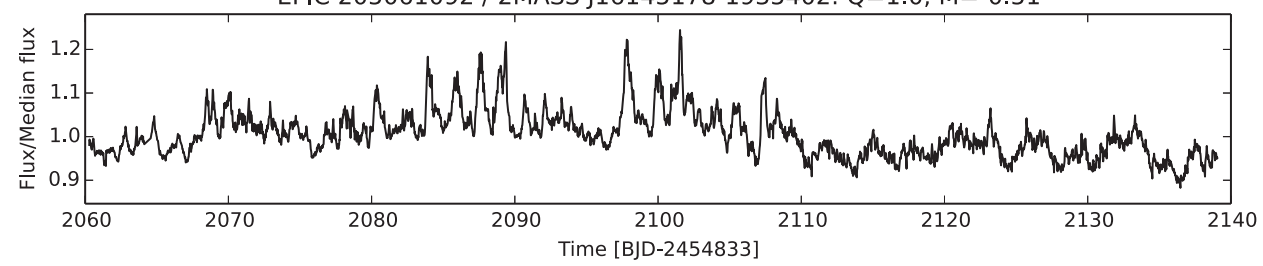

EPIC 204906020 / 2MASS J16070211-2019387: Q=0.93, $M=-0.47$

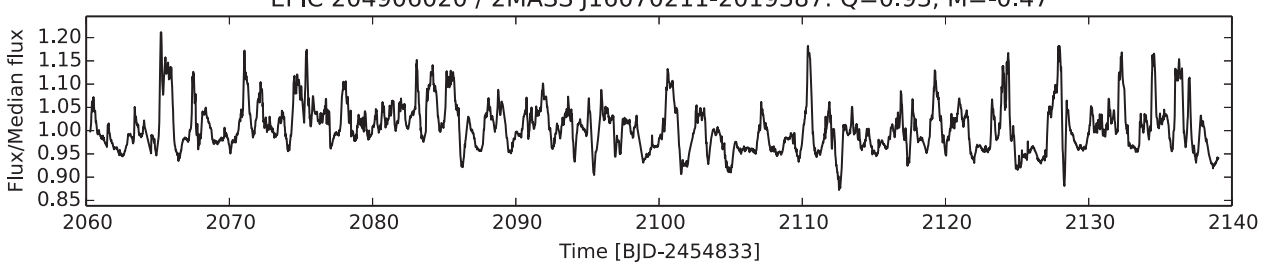

EPIC 203913804 / 2MASS J16275558-2426179: $Q=1.0, M=-0.37$

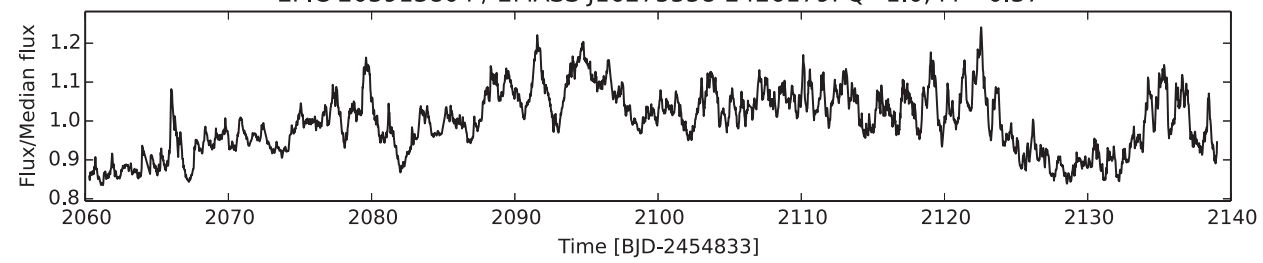

EPIC 203905625 / 2MASS J16284527-2428190: $Q=1.0, M=-0.31$

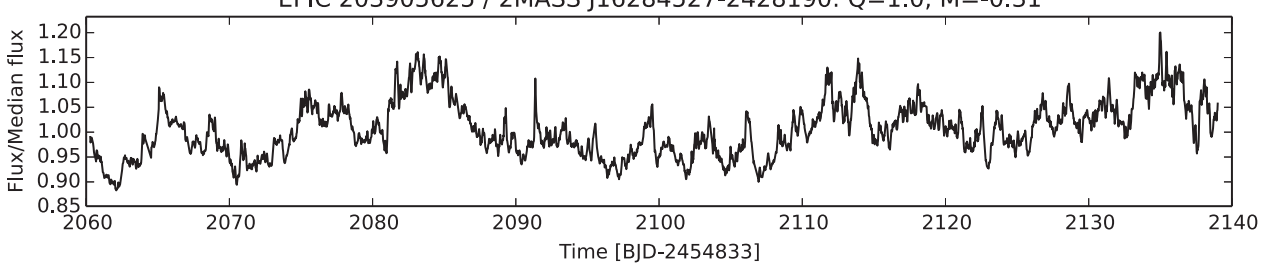

Figure 2. (Continued.)

they range from $\sim 10$ to over 400 for the most prominent burst events.

\section{Stellar Properties of the Accretion Burst Objects}

\subsection{Spatial Distribution}

The sample we have analyzed here is likely a mixture of ages from young $(<1-2 \mathrm{Myr})$ but still optically visible stars associated with the young $\rho$ Oph cloud, to somewhat older (5-10 Myr) stars in the off-cloud Upper Sco region, which still retain accreting circumstellar disks. Bursters as identified here make up $\sim 9 \%$ of the disk-bearing young star sample in the $K 2$ / C2 data set. We initially hypothesized that they would preferentially appear in the compact, young $\rho$ Oph cluster, as opposed to the more dispersed and older Upper Sco region. However, the spatial distribution shown in Figure 7 surprisingly reveals equal proportions of bursters in the two regions. Furthermore, there is no correlation with global extinction measures. This suggests that either the young population extends from $\rho$ Oph out to the surrounding areas, or that the burster phenomenon is less dependent on age than on disk properties such as mass. Erickson et al. (2011) found evidence for an intermediate age population ( $3 \mathrm{Myr}$ ) of YSOs outside of the main $\rho$ Oph cloud core, but within the main L 1688 cloud. Furthermore, Wilking et al. (2005) found a negligible age difference between sets of Upper Sco and L 1688 association members; both regions were estimated to be $\sim 3$ Myr old.

\subsection{Spectral Types}

To assess the mass distribution of stars displaying bursts, we have gathered spectral types from the literature. Those found for the bursters are displayed in Table 1. We have also selected a control sample of non-bursting stars from Luhman \& Mamajek (2012). That work presents spectral types for hundreds of USco members; we cull the list to include only non-bursting, inner disk-bearing stars observed in $K 2 \mathrm{Cam}-$ paign 2. The resulting set of 31 objects has spectral types ranging from $\mathrm{B} 8$ to M8, as shown in Figure 8. We compare this 

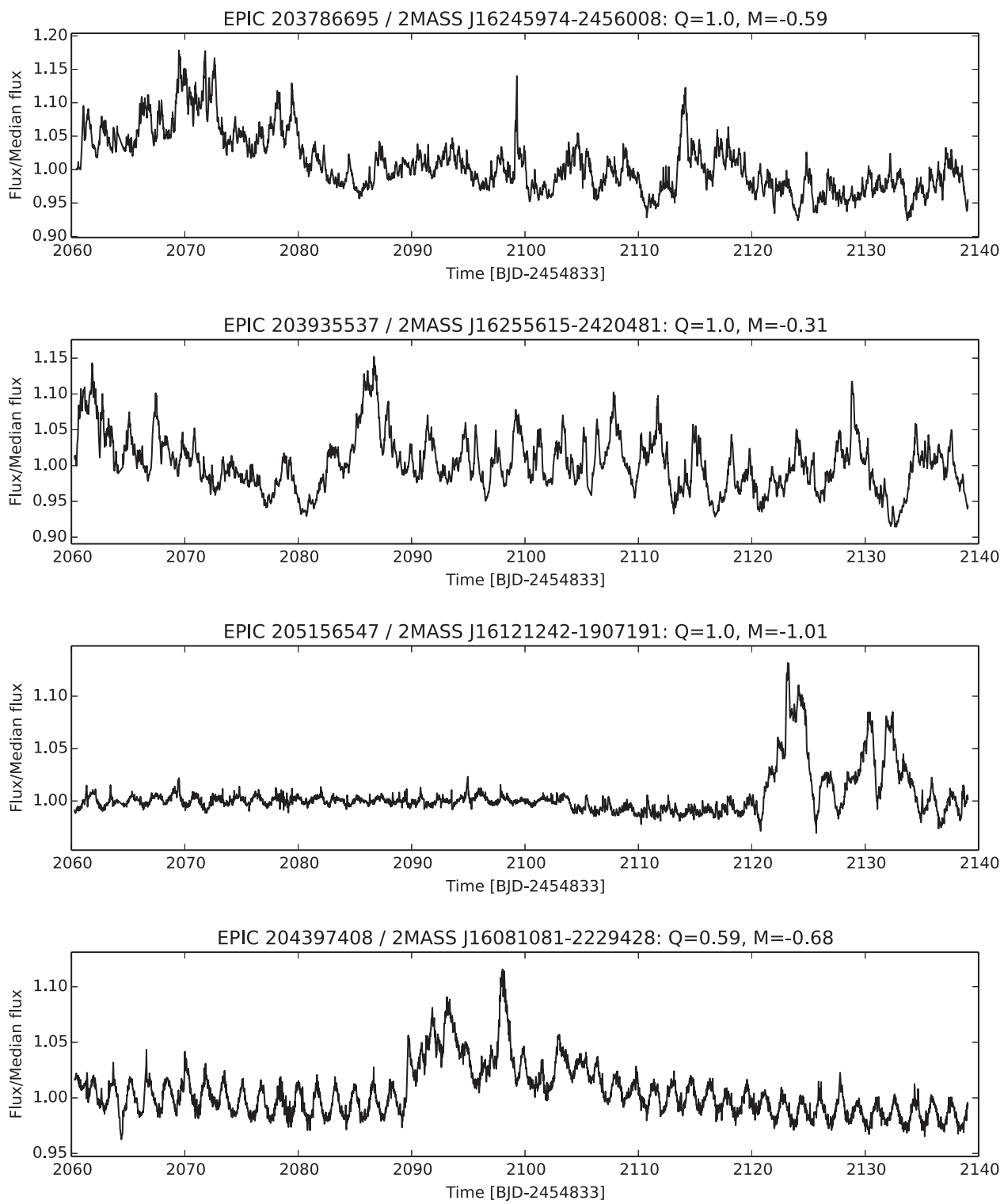

Figure 2. (Continued.)

against the distribution of spectral types for the bursters identified here, finding a significant difference only for the B-F spectral types. There are early-type stars in the sample but none of their variability is of the bursting type. $K 2$ light curves for these objects show mainly low-level quasi-periodic modulation. However, the spectral type distributions of bursters and non-bursters is very similar for the $\mathrm{K}-\mathrm{M}$ range. Statistically, the two sets are indistinguishable here. Thus we conclude that the preponderance of late-M spectral types among bursters is consistent with the increased fraction of young stars with disks at low masses.

\subsection{Multiplicity}

The presence of close stellar companions to the young stars in our sample may influence their variability properties. Indeed, it has been hypothesized that, for high-amplitude outbursting stars, such as FUors, events could be triggered by the presence of a perturbing companion (Lodato \& Clarke 2004; Reipurth \& Aspin 2004). However, support for this idea is mixed (Green et al. 2016). It is nevertheless worthwhile to check which, if any, of our burster sample may be in binary systems. We have vetted over half of the sample for multiplicity by examining the available high-resolution imaging as well as obtaining new speckle observations.

\subsubsection{Literature Assessment}

We mined the literature for adaptive optics and speckle imaging of these targets, and the details are tabulated as part of the individual object commentary in the Appendix. Only three objects have evidence for a close companion published in the literature:

1. EPIC 204906020 (2MASS J16070211-2019387),

2. EPIC 203786695 (2MASS J16245974-2456008),

3. EPIC 203905625 (2MASS J16284527-2428190). 

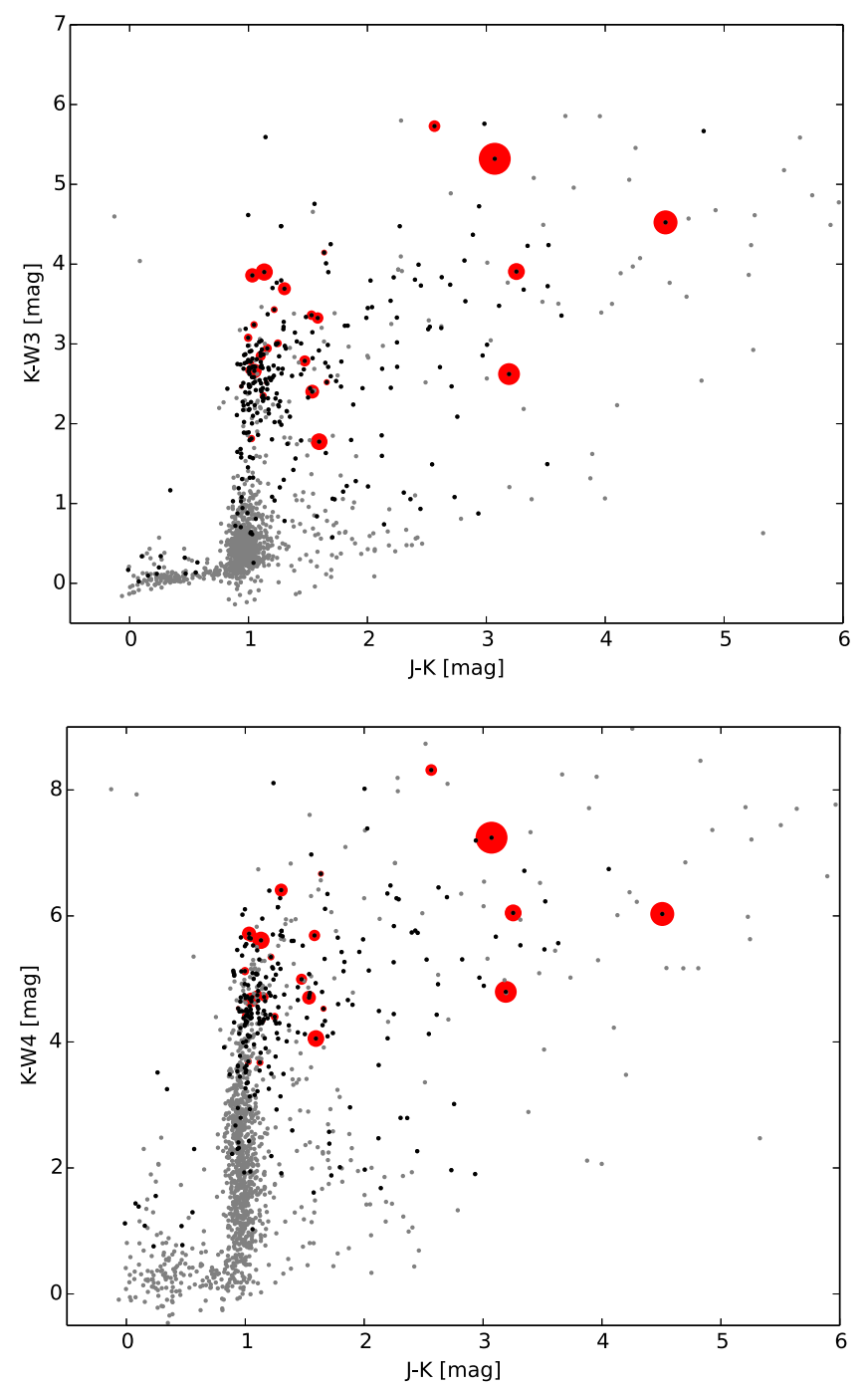

Figure 3. Near- and mid-infrared color-color diagram of stars in the Upper $\mathrm{Sco} / \rho$ Oph regions (gray), with disk-bearing stars in black and bursters highlighted in red. Burster point sizes are scaled by light-curve amplitude.

The first is a close binary (55 mas or 8 au; Kraus \& Hillenbrand 2012). Likewise, the second has a companion at $\sim 15$ au (100.4 mas; Koresko 2002). The last of these has an even closer companion at $\sim 2$ au (13 mas; Simon et al. 1995). Interestingly, all three systems also have wider ( $>50 \mathrm{au}$ ) companions. None of their light curves shows any hint of a periodicity. One additional object, EPIC 204342099 (2MASS J161534562242421), may have a 274 au separation companion (Gregorio-Hetem et al. 1992), but it is not clear whether the two stars are bound. EPIC 204830786 (2MASS J160757962040087 ) is associated with another star 21.15 away (>3000 au separation) but has not been surveyed for closer companions.

Other than these five objects, nine other bursters in our sample have been surveyed for multiplicity, at a variety of sensitivities and separations:

1. EPIC 204226548 (2MASS J15582981-2310077),

2. EPIC 203899786 (2MASS J16252434-2429442),

3. EPIC 203935537 (2MASS J16255615-2420481),

4. EPIC 203905576 (2MASS J16261886-2428196),

5. EPIC 203954898 (2MASS J16263682-2415518),

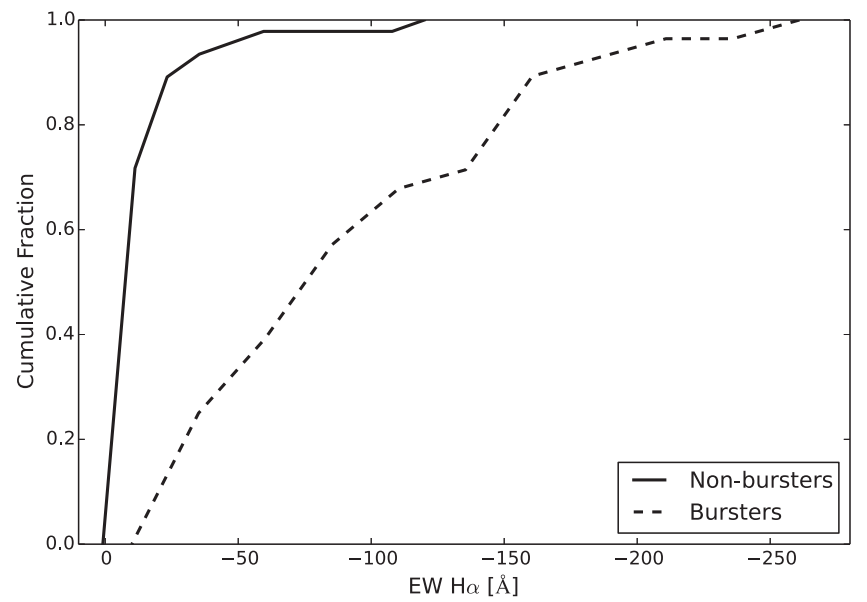

Figure 4. We show the cumulative distributions of $\mathrm{H} \alpha$ equivalent width for disk-bearing stars in our $K 2$ young star set with available spectroscopy; this set includes 28 bursters and 46 non-bursters. Where more than one $\mathrm{Ha}$ measurement is available, we adopt the mean value. The sample was binned in $10 \AA$ wide sets to produce the distribution. While the non-bursting stars tend to predominate between 0 and $-15 \AA$ (i.e., emission), the burster $\mathrm{H} \alpha$ values show a much wider dispersion, reaching much values up to $-250 \AA$. Thus the burst phenomenon seems to favor stars with high accretion rates. We note that there is a single disk-bearing star with quasi-periodic light curve that has a reported $\mathrm{H} \alpha$ equivalent width around $-120 \AA$. Such a value is highly unusual for a star with a non-bursting light curve.
6. EPIC 203822485 (2MASS J16272297-2448071),
7. EPIC 203913804 (2MASS J16275558-2426179),
8. EPIC 203928175 (2MASS J16282333-2422405),
9. EPIC 203794605 (2MASS J16302339-2454161).

No companions were found in these cases. However, the separations probed are very non-uniform and range from 10 mas $(1.5 \mathrm{au})$ in some cases to $1^{\prime \prime}-30^{\prime \prime}$ in others $(>150 \mathrm{au})$; details are provided in the Appendix.

\subsubsection{DSSI Targets}

In addition to data compiled from the literature, we also have speckle imaging observations of six bursters using DSSI (Section 2.3). For EPIC 204342099 (2MASS J161534562242421), we recover the companion reported at 1.9 by Gregorio-Hetem et al. (1992); however, we measure the separation to be 1 !. 50 ( $\sim 218 \mathrm{au})$, and a magnitude difference of $\Delta m=3.38$ at the $880 \mathrm{~nm}$ band. This star previously had direct imaging and aperture masking by Kraus \& Hillenbrand (2008) that ruled out further objects down to 240 mas (35 au). EPIC 204830786 (2MASS J16075796-2040087) has a previously noted possible companion at thousands of astronomical units of separation, but the DSSI observations otherwise support the hypothesis that this is a single star. EPIC 204 440603 (2MASS J16142312-2219338) has no previous multiplicity information, but the $880 \mathrm{~nm}$ image suggests a possible companion at a separation of 0 !' 1 . However, the lack of a similar detection at $692 \mathrm{~nm}$ and the faintness of this star makes speckle reconstruction challenging; thus the existence of such a companion remains indeterminate. EPIC 204360807 (2MASS J16215741-2238180) has no multiplicity information in literature, but we find it to be a 0 ". 48 separation binary (70 au), with $\Delta m=0.74$ at $880 \mathrm{~nm}$. EPIC 203935537 (2MASS J16255615-2420481) has no reported evidence for 

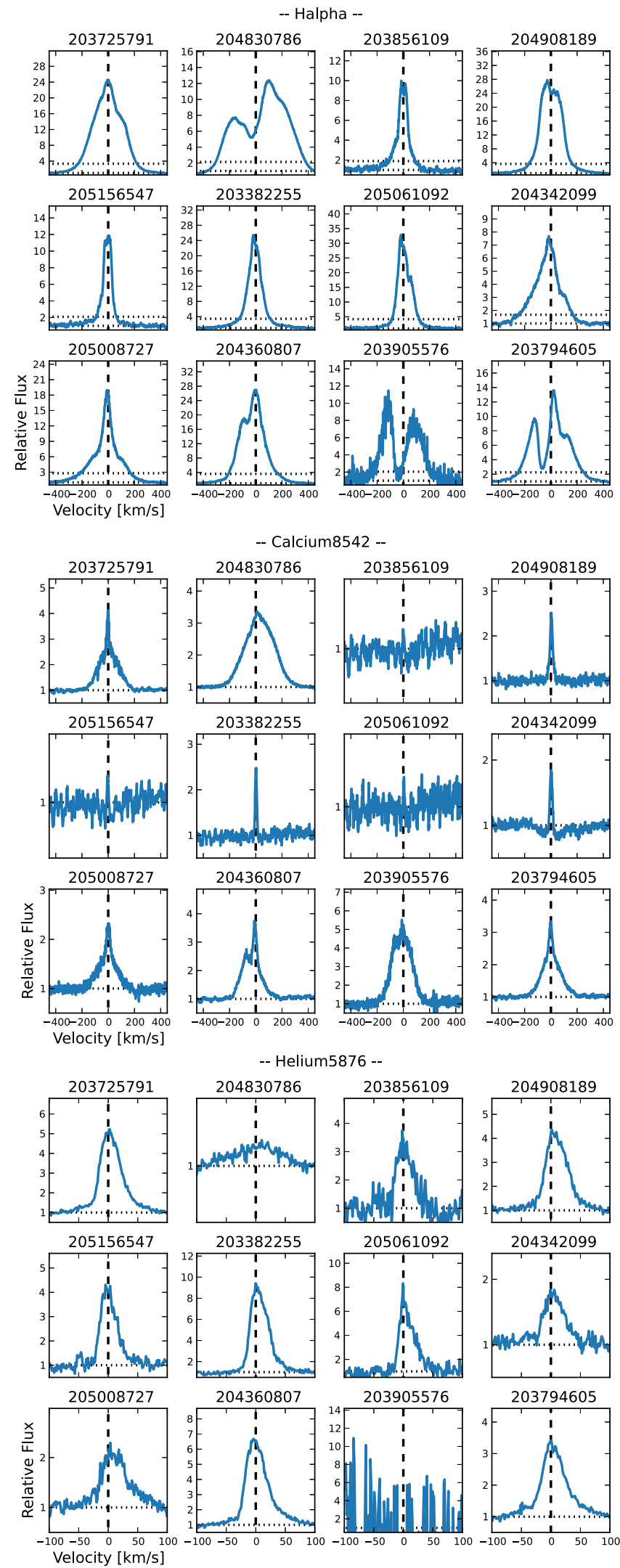

Figure 5. Line profiles in $\mathrm{H} \alpha$, Ca II $8542 \AA$, and He I $5876 \AA$ measured by Keck/HIRES for 12 of the 29 bursters, labeled by the EPIC identifier. The $\mathrm{H} \alpha$ panels include a horizontal line at $10 \%$ of the peak (un-normalized) flux in addition to the horizontal line indicating the continuum level. Note the change in velocity scale for the helium panels. Broad width and structured velocity profiles indicate accretion and wind phenomena.

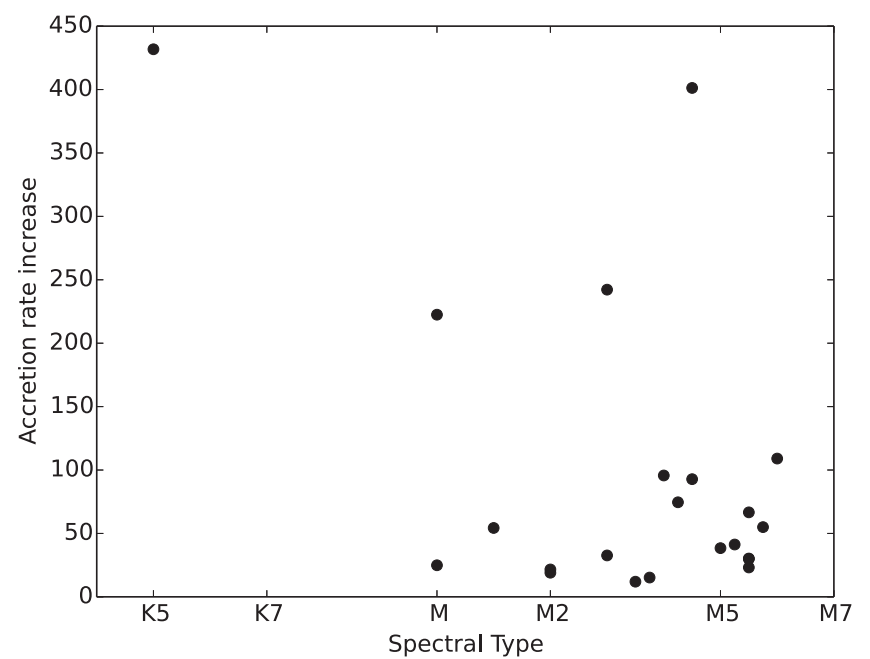

Figure 6. Estimated increase in accretion rate during bursts, as a function of stellar spectral type.

multiplicity, and we do not detect companions down to 0 !" 1 separation $(15 \mathrm{au})$ at $4-5 \mathrm{mag}$ of contrast in the 692 and $880 \mathrm{~nm}$ bands. Finally, EPIC 203928175 (2MASS J162823332422405) was reported by Cheetham et al. (2015) to host no companions down to 20 mas ( $3 \mathrm{au}$ ) at 1-3 mag contrast; our observations support the lack of binarity, with no detections outward of 0 ". 1 at $\Delta m \sim 4.4 \mathrm{mag}$.

\section{Time Domain Behavior}

In order to appreciate the diversity of the bursting behavior among our sample of objects, we must go beyond just the $M$ and $Q$ metrics discussed above. We quantified the peak-to-peak amplitudes of the bursters by first cleaning and normalizing the light curves and then computing the maximum-minus-minimum values. There are several ways to quantify light-curve timescales.

First, we measure the burst duty cycle, which is the fraction of time each object spends in a bursting state. This is by nature somewhat subjective because bursts display a range of amplitudes and shapes. We identified bursting portions of each light curve by first fitting and removing a low-order median trend to the light curve. This flattens the "continuum" level from which bursts arrive. We then measure the typical point-topoint scatter by shifting each point by one, subtracting from the original light curve, and dividing the standard deviation of the result by $\sqrt{2}$. Using this measure of scatter, we have found that burst behavior, as detected by-eye, includes points that lie about 15 times the scatter above the minimum of the continuum-flattened light curve. We thereby selected bursting and non-bursting sections for each time series. This method only failed for three objects (EPIC 204397408/2MASS J16081081-2229428, EPIC 205156547/2MASS J161212421907191, and EPIC 203856109/2MASS J16095198-2440197) that displayed intermittent quasi-periodic behavior that was picked up as bursts. We manually removed these light-curve portions for the statistical analysis. In Figure 9, we display the peak-to-peak amplitudes versus duty cycle of each burster. The duty cycles exhibit a large range of values, from almost $100 \%$ down to $\sim 10 \%$. Typically, the light curves with the highest amplitudes have higher duty cycles of $60 \%$ and above, with the 

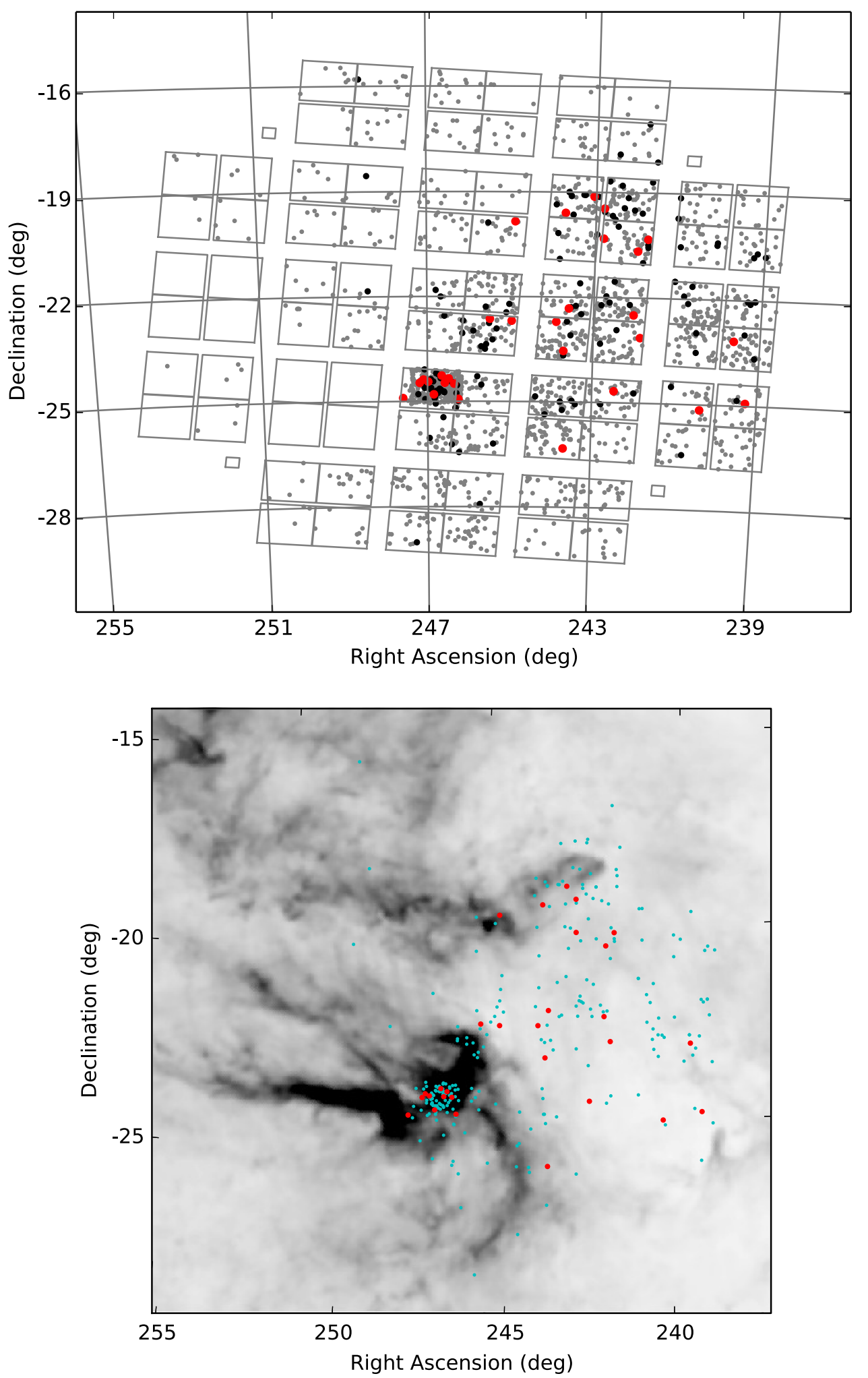

Figure 7. Top: spatial distribution of young stars (gray), including variables (black) and specifically bursters (red), overlaid on the $K 2$ field of view. The concentration of stars near R.A. $=246.8$, decl. $=-24.6$ is the $\rho$ Oph cluster. Bottom: young stars with disks (cyan) and bursters (red) overlaid on the Schlafly \& Finkbeiner (2011) extinction map of the $\rho$ Oph region.

exception of outlier EPIC 203954898/2MASS J162636822415518. This object may represent a distinct form of bursting behavior.

We also quantify the burst timescales by applying a method similar to the one described in Cody et al. (2014; Section 6.5 of that paper). In brief, this involves identifying peaks that rise above a particular amplitude level compared to the surrounding light curve. Once peaks are found, the median timescale separating them is computed. This procedure is repeated for a variety of amplitudes, from the noise level up to the maximum 


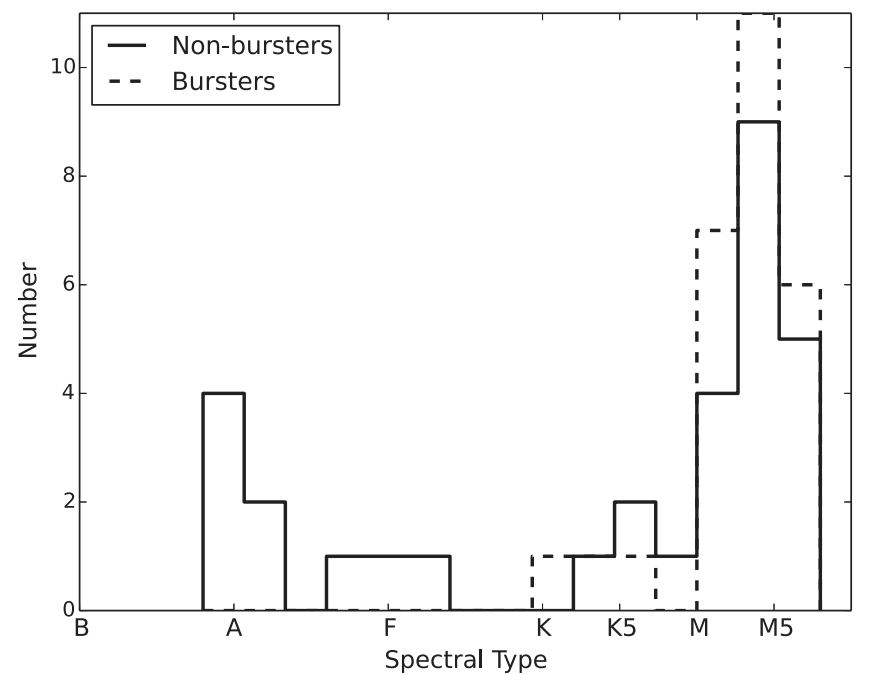

Figure 8. Spectral type distributions for bursting and non-bursting young diskbearing stars observed in $K 2$ Campaign 2 . Two distributions are very different for the $\mathrm{B}-\mathrm{F}$ range, but indistinguishable for $\mathrm{K}$ and $\mathrm{M}$ spectral types.

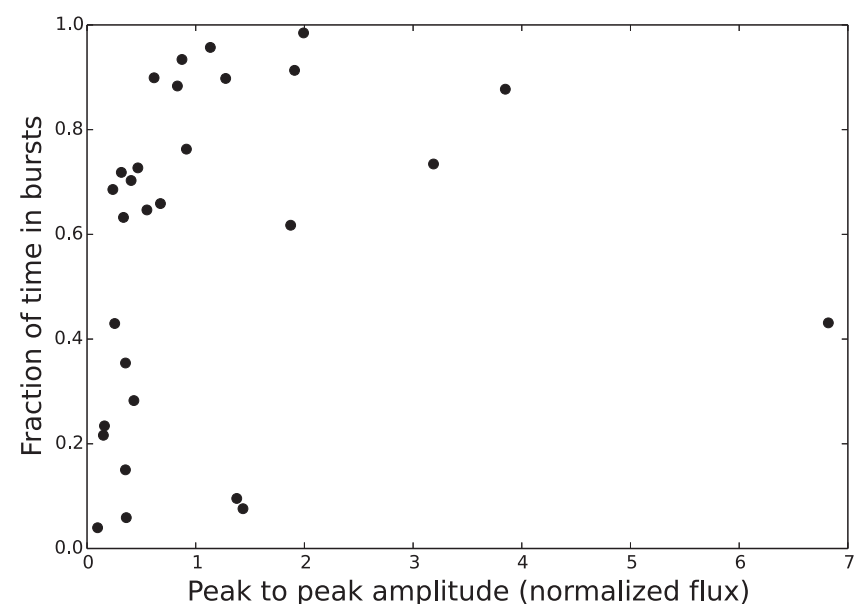

Figure 9. Maximum burst amplitude (in units of normalized flux) vs. duty cycle, i.e., fraction of time spent bursting.

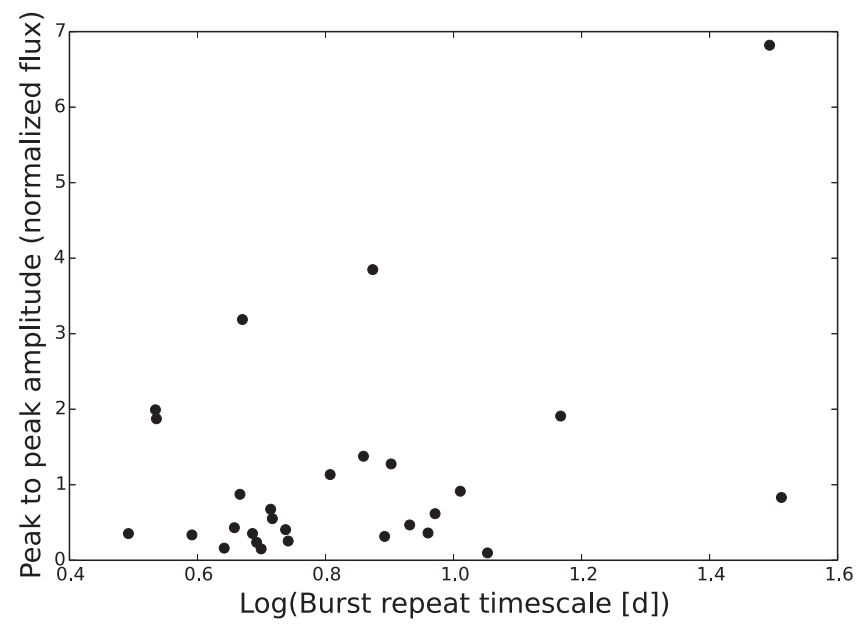

Figure 10. Burst repeat timescales vs. peak-to-peak light-curve amplitude in normalized flux.

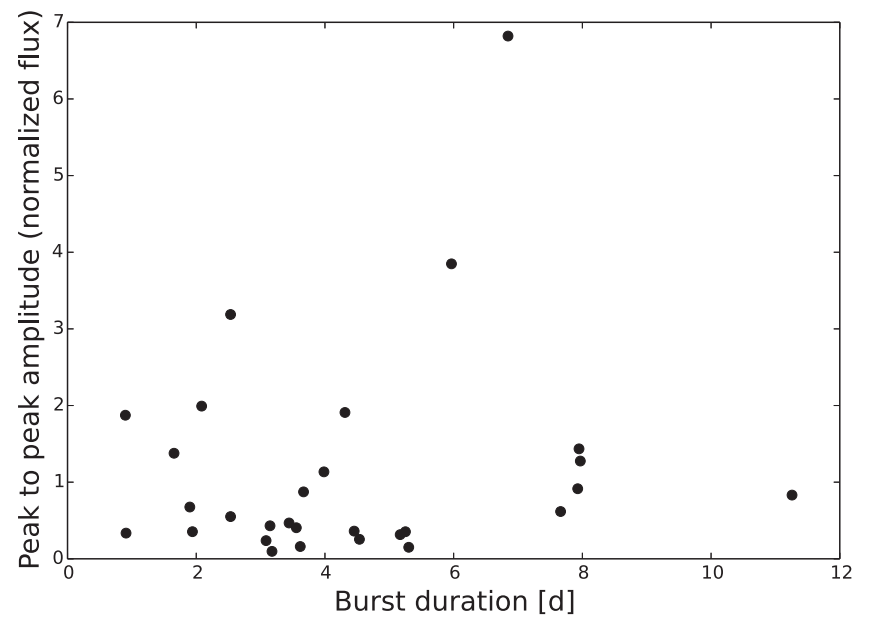

Figure 11. Burst durations vs. peak-to-peak light-curve amplitude in normalized flux.

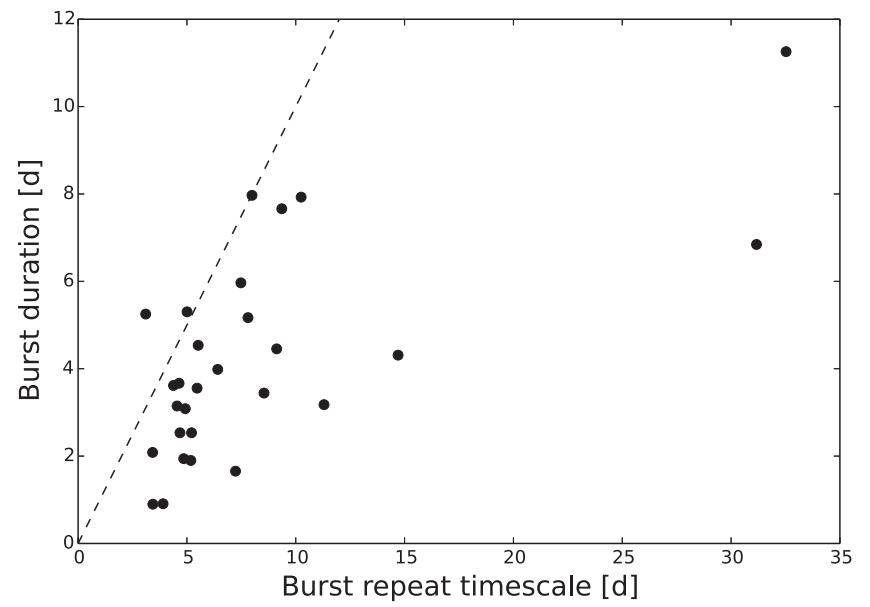

Figure 12. Burst repeat timescale vs. durations. The dashed line indicates where these two quantities are equal. We have left out EPIC 203382255 (2MASS J16144265-2619421) since it only has one burst and the repeat timescale is thus indeterminate.

light-curve extent. The result is a plot of timescale versus amplitude (e.g., Figure 32 in Cody et al. 2014). Finally, we take as a "representative" timescale the value corresponding to an amplitude that is $40 \%$ of the maximum peak-to-peak value (we note that this is different from the value of $70 \%$ adopted in Cody et al. 2014 and appears to be more appropriate for the burster light curves examined here). This computation only fails for the light curve of EPIC 203382255 (2MASS J161 44265-2619421), which displays only one burst event; here the timescale is indeterminate. In Figure 10, we display the peakto-peak amplitudes versus estimated timescale for each burster. Again, there is a large range of values, but no clear correlation with amplitude.

The burst duration is another way to quantify the observed events. This is a challenging measurement because there is a superposition of bursts with varying widths and heights. We simplify as above by only considering peaks that rise to a level of at least $40 \%$ of the maximum peak-to-peak value. For each peak, we identify the surrounding points that are more than 15 


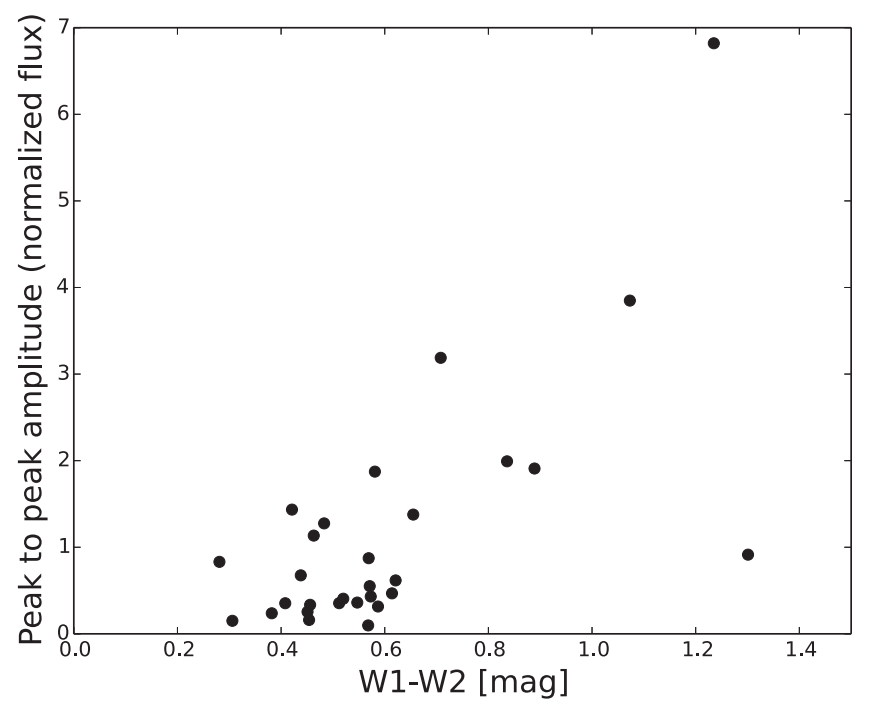

Figure 13. Peak-to-peak light-curve amplitude for bursters is shown against the $W 1-W 2$ color. All included objects have $W 1-W 2$ values indicative of inner disk dust, but the highest amplitude bursters tend to have larger infrared excesses, with one prominent exception (EPIC 205008727/ 2MASS J16193570-1950426; $W 1-W 2 \sim 1.3$ ).

times the point-to-point scatter above the minimum light-curve value (as was done for the burst duty cycle calculation). We then adopt the median burst duration of all such peaks in each light curve. The result is plotted against peak-to-peak amplitude in Figure 11. We also compare the durations with the repeat timescales in Figure 12. Here we find that burst duration is correlated with repeat timescale. This is somewhat expected since, by definition, a duration is typically larger than the average timescale between bursts. However, we observe a distinct lack of short bursts (duration $\leqslant 2$ days) with long repeat timescales. This may be rooted in the physical mechanism of the bursts.

We have also generated periodograms to identify any repeating components in the light curves. Most bursters do not exhibit periodicity but instead adhere to stochastic behavior, with any quasi-periodicity quantified via the $Q$ statistic (see Section 3 ). Those that do show significant periodicities (as indicated by $Q \leqslant 0.61$ and/or a strong, isolated periodogram peak) are EPIC 203794605/2MASS J16302339-2454161 ( $P=4.5$ days), EPIC 203899786/2MASS 16252434-2429442 ( $P=6.0$ days), EPIC 203928175/2MASS J16282333-2422405 ( $P=4.4$ days), EPIC 203954898/2MASS J16263682-2415518 ( $P=20.8$ days $)$, and EPIC 204347422/2MASS J16195140-2241266 ( $P=6.9$ days). The measured periods are similar to the burst repeat timescales inferred above. In addition, EPIC 203856109 (2MASS J16 095198-2440197), EPIC 204233955 (2MASS J16072955-230 8221), EPIC 204397408 (2MASS J16081081-2229428), and EPIC 205156547 (2MASS J16121242-1907191) display shorttimescale ( $P$ less than a few days) periodic behavior outside of their bursting states. These periods are more typical of the $K 2 \rho$ Oph and Upper Sco sample as a whole (see L. M. Rebull 2017, in preparation) and likely measure stellar rotation, whereas those of the bursts are longer by factors of at least several.

Few of the timescale metrics show any relation to [circum] stellar properties, but one potential correlation stands out in peak-to-peak amplitude versus the infrared $W 1-W 2$ color (Figure 13), which is indicative of a dusty inner disk. These two quantities are correlated at a significance level of $4 \times 10^{-5}$ (Pearson $r$ coefficient of 0.69). This is also borne out in Figure 3, which suggests that the dustiest objects have the highest light-curve amplitudes.

\section{Discussion and Summary}

$K 2$ data from Campaign 2 have probed the optical burst properties of young stars on timescales ranging from hours up to several months, with approximately $8 \%-10 \%( \pm 2 \%)$ of strong disk sources exhibiting burst behavior. This is roughly in agreement with the $13_{-2}^{+3} \%$ fraction found for NGC 2264 (Cody et al. 2014; Stauffer et al. 2014). It is possible that an even larger fraction of young stars undergo bursting, but are not detected as such if the amplitude is low (i.e., $<100 \%$ peak to peak). Burst behavior could in some cases be masked by other types of variability.

Burster stars host inner circumstellar disks, as evidenced by WISE excesses and SEDs (Figure 14). Furthermore, there is a positive correlation between the $W 1-W 2$ color and lightcurve peak-to-peak amplitude. Stronger inner disk excesses appear to be associated with bigger bursts. Most of these objects have exceptionally strong $\mathrm{H} \alpha$ emission, as well as other Balmer lines, He I, and $\mathrm{Ca}$ II in emission, as is typical for strongly accreting stars. Viewing geometry may play a role in setting the observability of the bursting phenomenon, but thus far we only have disk inclination constraints from ALMA on two sources in the sample, both of which are tilted at $\sim 42^{\circ} \pm 15^{\circ}$.

Members of the bursting sample typically exhibit multiple discrete brightening events, some lasting up to or just over one week. Time domain properties of the bursters are diverse, with some stars exhibiting a nearly continuous series of bursts and others displaying one or two isolated episodes superimposed on otherwise lower amplitude or quasi-periodic behavior. The majority of these light curves show flux variations of less than a factor of two and are similar to the objects in the $\sim 3 \mathrm{Myr}$ NGC 2264 highlighted by Stauffer et al. (2014). However, 7 of 29 bursters display discrete brightenings of more than $100 \%$ on day to week timescales, unlike most heretofore classified young stellar variables that we are aware of. Of particular interest is the subset of these for which the bursts repeat quasiperiodically: EPIC 204347422 (2MASS J16195140-2241266), EPIC 203928175 (2MASS J16282333-2422405), and EPIC 203954898 (2MASS J16263682-2415518). It is unclear as to what physical phenomenon sets the periods of 6.9 days, 4.4 days, and 20.8 days (respectively) in these cases; it appears to be relatively independent of stellar mass, as indicated by spectral type. There is also a lack of evidence for binarity in most of the bursters, though in some cases limits from imaging are relatively shallow. We thus speculate that burst events are not triggered by any companion, but rather by a repetitive interaction between the stellar magnetic field and inner disk.

From the theoretical perspective, magnetically channeled accretion is not predicted as steady in numerical simulations. An inner disk is truncated at a balance point between the inward pressure from accretion and the outward pressure of the magnetosphere. This produces variable and possibly cyclic mass flow due to instabilities and pulsational behavior. Such variations in the mass loading of accretion columns are modeled under different physical scenarios by, e.g., Lovelace 
et al. (1995), Goodson \& Winglee (1999), Romanova et al. (2002, 2004, 2005), and D'Angelo \& Spruit (2010, 2012).

The disk corotation radius $\left(r_{c}\right)$ and the magnetospheric radius $\left(r_{m}\right)$ are critical in determining the regime of accretion under which a star-disk system falls. When $r_{m}<r_{c}$, gas at the inner disk edge rotates faster than the star and its magnetosphere, causing it to flow along magnetic field lines onto the central star. As long as $r_{m}>0.7 r_{c}$, "stable" accretion occurs (Blinova et al. 2016) and gas follows two funnel streams onto the star (Romanova et al. 2003b, 2004). For smaller values of $r_{m}$, the Rayleigh-Taylor instability sets in and accretion becomes chaotic, with many tongues of matter extending from the inner disk to the stellar surface (Kulkarni \& Romanova 2008; Romanova et al. 2008). Numerous hot spots are present on the stellar surface, and the associated light curves display irregular bursting. This regime may be responsible for the large subset of bursters that we observe with high duty cycles.

When $r_{m}$ is up to a factor of two larger than $r_{c}$, on the other hand, gas in the inner disk rotates slower than the magnetosphere and is accelerated azimuthally. Models (e.g., D'Angelo \& Spruit 2012) predict a "trapped disk" regime in which relatively continuous accretion bursts occur on short timescales. When $r_{m} \gg r_{c}$, material tends to be flung out azimuthally in what is known as the "strong propeller" regime (Romanova et al. 2003a). The light curves of several of our objects (EPIC 203954898/2MASS JJ16263682-2415518 and EPIC 204347422/2MASS J16195140-2241266) strongly resemble the simulated mass flow variations predicted by Zanni \& Ferreira (2013) and Lii et al. (2014) for propeller behavior. In this scenario, the matter accretes episodically in three phases: first, material accumulates at the inner disk boundary, unable to flow inward. The magnetosphere is compressed toward the stellar surface. Next, compression reaches the point at which the magnetosphere can no longer withstand the gravitational forces on the accumulated material; gas accretes rapidly onto the star in a funnel flow. Finally, the compression pressure is relieved, accretion ceases, and the magnetosphere is able to expand outward again. Following this sequence, the cycle repeats. The time between bursts is longer than in other regimes, and the accretion rate can follow a flarelike pattern (rapid rise; slower decline) as a function of time. Strong outflows may also be present. EPIC 203382255 (2MASS 16144265-2619421) shows outflow signatures and also has the longest repeat timescale, making it an additional propeller regime candidate. In contrast, a few other objects with outflow-related spectral lines do not display the expected alternating burst and quiescence pattern.

Further investigation of these targets is necessary to estimate magnetic and corotation radii and compare with the theoretical expectations. The ratio $r_{m} / r_{c}$ depends on mass accretion rate, magnetic field strength, and stellar spin period-parameters that we are unable to determine independently for this sample of burster stars. In the meantime, the burst timescales measured for our sample provide perhaps the best indication of the physical mechanisms at work. Duty cycles range from $10 \%$ and all the way up to nearly $100 \%$ - suggesting that we are seeing different modes of accretion from continuous to episodic and less frequent. The possible correlation between burst duration and repeat timescale (Figure 12) may imply a relationship between mass loading timescale and accretion rate.

A major open question stemming from this work is what the origin of the day to (multi-)week repeat timescales that we observe is, and how it relates to young outbursting stars with much longer duty cycles (e.g., EXors and FUors). Prominent examples from the literature include V899 Mon, which has had repeated bursts separated by $\sim 1$ year (Ninan et al. 2015), and V1647 Ori, which repeats at $\sim 2$ years (Aspin \& Reipurth 2009). On even longer timescales, other EXor type stars tend to outburst once or twice per decade. Unlike the $K 2$ objects, the longer among these timescales are thought to be related to the viscous timescale in the disk. Here, the material drains inward and must undergo replenishment before the next instability-driven episode can occur. It has been speculated that the frequency and amplitude of outbursts may be set by the accretion rate, with younger and higher amplitude outbursting objects accreting more rapidly $\left(\dot{M} \sim 10^{-7}\right.$ to a few $10^{-4} M_{\odot} \mathrm{yr}^{-1}$; Audard et al. 2014, p. 387). We would then expect accretion rates for our own sources to be somewhat lower. This does indeed seem to be the case, as the median accretion rate where available for bursters is $10^{-8.1} M_{\odot} \mathrm{yr}^{-1}$ (for exact values, see the notes on individual objects in the Appendix). We have found (Section 4.2) that this increases one to two orders of magnitude during the most extreme $K 2$ light-curve peaks. Further spectroscopic measurements during times of definitive bursting may confirm these estimates.

In summary, the $K 2$ mission is providing an unprecedented view of the time domain properties of young stars, and showing that the optical photometric manifestations of accretion phenomena take on a wide variety of timescales and amplitudes. Followup observations, including spectroscopy, should be carried out to investigate changes in spectral emission and inner disk structure in the highest amplitude objects presented here.

The work of AMC was supported by a NASA/NPP fellowship. We thank the referee for useful feedback that improved this paper. We acknowledge Luisa Rebull for calling our attention to one of the burst type objects that we had overlooked in our initial examination. Thanks also to Nic Scott for assistance with DSSI observations on the Gemini South telescope.

This paper includes data collected by the $K 2$ mission. Funding for the $K 2$ mission is provided by the NASA Science Mission directorate. The spectroscopic data were obtained at the W.M. Keck Observatory, which is operated as a scientific partnership among the California Institute of Technology, the University of California and the National Aeronautics and Space Administration. The Observatory was made possible by the generous financial support of the W.M. Keck Foundation. These results are also based on observations obtained as part of the program GS-2016A-Q64 at the Gemini Observatory, which is operated by the Association of Universities for Research in Astronomy, Inc., under a cooperative agreement with the NSF on behalf of the Gemini partnership: the National Science Foundation (United States), the National Research Council (Canada), CONICYT (Chile), Ministerio de Ciencia, Tecnología e Innovación Productiva (Argentina), and Ministério da Ciência, Tecnologia e Inovação (Brazil).

This paper has utilized the SIMBAD and Vizier services, through which data from USNO-B, APASS, 2MASS, and WISE were collected. 


\section{Appendix A Notes on Individual Objects}

Not all of our sample stars appear in the literature, but for those that have been studied previously, we highlight the key results here. We also incorporate information from our new speckle and spectroscopic observations.

\section{A.1. EPIC 203382255/2MASS 16144265-2619421}

This source is cataloged by Lodieu (2013) as an astrometric and photometric member, but is not otherwise studied in the literature. Our spectra suggest a spectral type of M4-M5.5 depending on the spectral range (earlier at bluer wavelengths). Lithium is present with $W_{\mathrm{Li}}=0.30 \mathrm{~A}$ along with the $\mathrm{H} \alpha$ emission reported in Table 1 , $\mathrm{He}$ I, weak $\mathrm{NaD}$, and $\mathrm{Ca}$ II are seen in emission. Broad [O I] is also present. The system is a clear accretion/outflow source.

\section{A.2. EPIC 203725791/2MASS J16012902-2509069}

This star was first proposed as an Upper Sco member by Ardila et al. (2000), based on RIZ photometry. It was only recently confirmed by Rizzuto et al. (2015), based on significant lithium absorption. No other data has been reported for this star.

Our HIRES spectrum indicates a spectral type of M2 with lithium absorption strength $W_{\mathrm{Li}}=0.3 \AA$. Strong $\mathrm{H} \alpha$ emission is seen, as indicated in Table 1, along with lorentz-broadened He I. NaD, weak narrow Fe II as well as Mg I emission, twocomponent $\mathrm{Ca}$ II, and $\mathrm{O} \mathrm{I}$ emission. Very weak [O I] is also exhibited.

\section{A.3. EPIC 203786695/2MASS J16245974-2456008}

This star, WSB 18, was first noted as part of Wilking et al.'s (1987) $\mathrm{H} \alpha$ emission survey of the the $\rho$ Ophiuchi complex. Lithium absorption was detected by Erickson et al. (2011), confirming youth. It is a visual binary with a separation of 1"! 1 (138 au) and a 0.49 flux ratio (Reipurth \& Zinnecker 1993). Therefore, both components contribute to our $K 2$ light curve. According to Brandner \& Zinnecker (1997), the primary has a spectral type of M2, whereas the secondary is M2.5. Both show $\mathrm{H} \alpha$ in emission, but the primary does not appear to have a disk (McCabe et al. 2006), whereas the secondary does. Furthermore, Koresko (2002) found that the primary itself is a double star with 0 "! 1 separation.

No detections of these sources at $>70 \mu \mathrm{m}$ were made with Herschel. In the submillimeter, Andrews \& Williams (2007) put an upper limit of $0.003 M_{\odot}$ on the dust mass surrounding this source, though it is unclear which components were included.

\section{A.4. EPIC 203794605/2MASS J16302339-2454161}

According to Wilking et al. (1987), this star displayed an $\mathrm{H} \alpha$ emission intensity of 3 on a scale of $1-5$ (where 1 is weak and 5 is strong). Simon et al. (1995) performed a direct imaging search for companions in the 0. " $005-10^{\prime \prime}$ separation range, but did not find anything. Nor were any companions detected in the Catalog of High Angular Resolution Measurements (CHARM; Richichi \& Percheron 2002), its successor CHARM2 (Richichi et al. 2005), or the Ratzka et al. (2005) speckle imaging survey of $\rho$ Oph. For the latter, no companions were detected down to
0.04 (0.14) times the stellar brightness at 0. ! 5 (0."15) separation. This star was included in the Spitzer c2d legacy survey.

Our HIRES spectrum is veiled, but consistent with a spectral type of M3.5-M5. Lithium is present with strength $W_{\mathrm{Li}}=0.32 \AA$. $\mathrm{H} \alpha$ emission as reported in Table 1 is strong with several components and a blueward-displaced central absorption. Additional emission includes He I with lorentzian wings, broad $\mathrm{NaD}$, many multi-component Fe II lines, strong Ca II with multiple component and O I 8446. Among the outflow lines, only multi-component [O I] is seen.

\section{A.5. EPIC 203822485/2MASS J16272297-2448071}

This star, WSB 49, is in the $\rho$ Ophiuchus cluster. It was first detected as part of Wilking et al.'s (1987) $\mathrm{H} \alpha$ emission line survey. It is also an IRAS source (Wilking et al. 1989). It was first discovered as an X-ray source with the ROSAT High Resolution Imager (Grosso et al. 2000), and also noted XMM Newton observations by Pillitteri et al. (2010). Wilking et al. (2005) confirmed its youth via detection of lithium absorption. Erickson et al. (2011) estimate a mass of $0.17 M_{\odot}$. The star was surveyed for multiplicity, but no companions were detected down to $>0$ "! 15 separation at flux ratios of 0.06 .

An infrared excess is detected with Spitzer (Evans et al. 2009). Andrews \& Williams (2007) observed it in the submillimeter, classifying the disk as class II, with $<0.005 M_{\odot}$ of material. In addition, the disk is detected at $70 \mu \mathrm{m}$ with Herschel/PACS (Rebollido et al. 2015). It was listed as a long-timescale near-infrared variable by Parks et al. (2014), while Günther et al. (2014) detected $20 \%$ variations in the mid-infrared with Spitzer.

\section{A.6. EPIC 203856109/2MASS J16095198-2440197}

There is no previous literature on this source. Our spectrum indicates a spectral type of M5-M5.5 with lithium at $W_{\mathrm{Li}}=0.61 \AA$. Moderate $\mathrm{H} \alpha$ emission, as indicated in Table 1 , is present and has a multi-component profile. Weak, also multicomponent, $\mathrm{He} \mathrm{I}$ is also present, but no other emission lines.

\section{A.7. EPIC 203899786/2MASS J16252434-2429442}

This star in the $\rho$ Oph cluster is also known as V852 Oph and SR 22. Its emission line spectrum was reported as early as the mid-20th century (Struve \& Rudkjøbing 1949), with prominent hydrogen, Ca II, and Fe II noted. Herbig \& Kameswara Rao (1972) and Herbig \& Bell (1988) included it in their emission line star catalogs. It is a ROSAT X-ray source (Casanova et al. 1995). Irregular variability was also reported early on by Satyvaldiev (1972) and Filin \& Satyvoldiev (1975).

Andre \& Montmerle (1994) label the object as class III based on its $2.2-10 \mu \mathrm{m}$ slope, and despite its previous classification as a classical T Tauri star by Cohen \& Kuhi (1979). It was relabeled as class II by Greene \& Lada (1996), based on their 1.1-2.4 $\mu \mathrm{m}$ survey. Andrews \& Williams (2007) confirmed the disk with submillimeter observations, detecting $0.002 M_{\odot}$ of material. Similarly, Mohanty et al. (2013) report $2.05 M_{\text {Jup }}$ of material, based on SCUBA-2 $850 \mu \mathrm{m}$ observations. Rebollido et al. (2015) detected the disk at $70 \mu \mathrm{m}$ with Herschel, and reported that there is a gap in the spectral energy distribution that suggests a hole. 
The source is evidently a single star. Ratzka et al. (2005) ruled out any companions down to a flux ratio of 0.07 at a separation of 0 !' 15 , and Greene \& Lada (1996) did not detect any radial velocity variations indicative of a spectroscopic binary. Cheetham et al. (2015) reported no companions down to 10 mas and contrasts of several magnitudes.

\section{A.8. EPIC 203905576/2MASS J16261886-2428196}

This star-better known as VSSG 1-boasts a disk that was first detected by IRAS (Clark 1991). The mid-infrared slope, $\alpha$, is -0.4 , making it a class II disk according to Andre \& Montmerle (1994; though Wilking et al. 1989 earlier classified it as class I). McClure et al. (2010) used SPEX to measure an $n_{2-25}$ spectral index of -1.26 , confirming the class II categorization. They also reported a small $10 \mu \mathrm{m}$ silicate feature. Andrews et al. (2010) have observed this source's disk with the Submillimeter Array at $0.87 \mathrm{~mm}$ and estimated a dust mass of $0.029 M_{\odot}$, along with an accretion rate of $10^{-7} M_{\odot}$ $\mathrm{yr}^{-1} \cdot \mathrm{H}_{2} \mathrm{O}$ was detected in the Spitzer IRS spectrum obtained by Pontoppidan et al. (2010), along with $\mathrm{HCN}, \mathrm{C}_{2} \mathrm{H}_{2}$, and $\mathrm{CO}_{2}$. Salyk et al. (2011) confirm these detections and estimate a disk mass of $0.029 M_{\odot}$. They infer a disk inclination of $53^{\circ}$. Submillimeter observations with ATCA (Ricci et al. 2010) resulted in an estimated disk outer radius of 100-300 au and a much lower dust mass of $4.5 \times 10^{-5}-1.9 \times 10^{-4} M_{\odot}$. Antoniucci et al. (2014) looked for mid-infrared variability in this source and concluded that it is not an EXor candidate. Natta et al. (2006) report a fairly high accretion rate of $10^{-7.19} M_{\odot}$ $\mathrm{yr}^{-1}$ and $\mathrm{a} \mathrm{Pa} \beta \mathrm{EW}$ of $8.9 \AA$ in emission. According to the work of Ratzka et al. (2005), no companions are visible down to a flux ratio of 0.04 at a separation of 0 ". 15 .

Our spectrum exhibits strong $\mathrm{H} \alpha$ as indicated in Table 1 with a double-peaked broad profile, as well as strong and broad Ca II triplet as well as O I 8446 emission. There is very little in the way of absorption, presumably due to heavy veiling, and spectral types from $K 7$ to mid-M are plausible. For the same reason, aggravated by low signal-to-noise in this region of the spectrum, there is no lithium measurement.

\section{A.9. EPIC 203905625/2MASS J16284527-2428190}

EPIC 203905625, also known as V853 Oph and SR 13, is a late-type star in $\rho$ Ophiuchus with reported spectral types from M2 to M4 (Wilking et al. 2005). Accretion signatures include $\mathrm{H} \alpha$ as well as calcium in emission. Rydgren et al. (1976) first reported strong veiling in the star's spectrum. Bouvier \& Appenzeller (1992) found that the $\mathrm{H} \alpha$ emission is variable (30-48 $\AA$ ). Natta et al. (2006) estimated an accretion rate of $10^{-8.31} M_{\odot} \mathrm{yr}^{-1}$, from near-infrared spectra. They detected $\mathrm{Pa} \beta$ in emission, at an EW of $-1.7 \AA$.

$\mathrm{X}$-rays from this object were first detected with Einstein (Montmerle et al. 1983). A disk around this star was also observed, with IRAS (Clark 1991). Andre \& Montmerle (1994) used $1.3 \mathrm{~mm}$ observations to detect the disk; they classify it as class II, based on a $2.2-10 \mu \mathrm{m}$ slope of -0.8 . Pontoppidan et al. (2010) detect $\mathrm{H}_{2} \mathrm{O}, \mathrm{OH}, \mathrm{HCN}$, and $\mathrm{C}_{2} \mathrm{H}_{2}$ in a Spitzer IRS spectrum of this target. Rebollido et al. (2015) report Herschel detections at 70 through $500 \mu \mathrm{m}$; they classify the system as transitional, based on 12-24 $\mu \mathrm{m}$ data.

The star is a multiple system, with a companion first detected at 0."4 separation via speckle imaging (Ghez et al. 1993). Aspin et al. (1997) and Ratzka et al. (2005) confirmed the 0." 4 and a 0.238 flux ratio. Simon et al. (1995) conducted an IR imaging survey that revealed a closer companion at a separation of 13 mas. McCabe et al. (2006) found that both the primary and its 0." 4 companion have class II disks. Andrews \& Williams (2007) measured a total disk mass of $0.01 M_{\odot}$ using submillimeter observations, while Mohanty et al. (2013) measured a similar 7.81 $M_{J}$ from SCUBA-2 $850 \mu \mathrm{m}$ observations.

Variability in this object was initially reported by Rydgren et al. (1976). Satyvoldiev (1982) found optical variations from 12.6 to 14.7. Herbst et al. (1994) observed $V$-magnitude fluctuations from 12.83 to 13.52 , while Grankin et al. (2007) reported $V$ magnitudes between 12.61 and 13.87. Likewise, Shevchenko \& Herbst (1998) conducted photometric monitoring, reporting a $V$-band amplitude of $0.91 \mathrm{mag}$. The light curves are too sparsely sampled to identify any morphological features, although they are classified as "irregular." The broadband $K 2$ light curve exhibits 0.2 mag events, suggesting that amplitudes are higher at bluer wavelengths. This is confirmed by Herbst et al. (1994)s estimate of $d U / d V: 2.39$.

\section{A.10. EPIC 203913804/2MASS J16275558-2426179}

This target is also known as SR 10 and V2059 Oph. It originally appeared in Herbig \& Kameswara Rao's (1972) catalog of emission line stars. Both Satyvaldiev (1972) and Kukarkin et al. (1977) list it among their variable star compilations; Satyvoldiev (1982) labeled the variations as "irregular."

$\mathrm{H} \alpha$ emission at $40 \AA$ was reported early on by Rydgren (1980). Appenzeller et al. (1983) also observed strong $\mathrm{H} \alpha$, as well as He I and Fe II in emission. Wilking et al. (1987) noted this star in their $\mathrm{H} \alpha$ emission survey, and Wilking et al. (2005) again measured $\mathrm{H} \alpha$ in emission as well as lithium in absorption. Natta et al. (2006) estimated the accretion rate to be $10^{-7.95} M_{\odot} \mathrm{yr}^{-1}$; they detected $\mathrm{Pa} \beta$ emission at an $\mathrm{EW}$ of $-5.6 \AA$. Najita et al. (2015) report a slightly lower accretion rate of $10^{-8.3} M_{\odot} \mathrm{yr}^{-1}$.

Simon et al. (1995) conducted an imaging survey for binary companions at project separations from 0 ". 005 to $10^{\prime \prime}$ down to a $K$ magnitude of 11.1. Likewise, Ratzka et al. (2005) searched for companions at separations of 0 ." 15 and 0 ". 50 but did not identify any down to flux ratios of 0.04 and 0.02 , respectively. Richichi \& Percheron (2002), Richichi et al. (2005), Barsony et al. (2003), and Cheetham et al. (2015) did not detect companions either, down to 10-20 mas separation at several magnitudes contrast. Thus this object appears to be a single star.

EPIC 203913804/2MASS J16275558-2426179 is a ROSAT X-ray source (Casanova et al. 1995). The star is also an IRAS source (Wilking et al. 1989). Andre \& Montmerle (1994) identified a class II circumstellar disk, and Gutermuth et al. (2009) later confirmed with Spitzer data. Andrews \& Williams (2007) studied the system in the submillimeter and deduced an upper limit on the disk mass of $0.005 M_{\odot}$, based on the $1.3 \mathrm{~mm}$ flux. Similarly, Mohanty et al. (2013) found an upper limit of $5.0284 M_{\text {Jup }}$. Rebollido et al. (2015) detected the disk at $70 \mu \mathrm{m}$ with Herschel.

\section{A.11. EPIC 203928175/2MASS J16282333-2422405}

EPIC 203928175 is a star in the $\rho$ Ophiuchus region that is also known as SR 20 W (Struve \& Rudkjøbing 1949) and 
ROXC J162823.4. Wilking et al. (2005) report a spectral type of $\mathrm{K} 5$, along with variable $\mathrm{H} \alpha$ emission and an $\mathrm{EW}$ of $35 \AA$.

It was surveyed for binarity by Ratzka et al. (2005), but no companions were detected down to 0.08 times the stellar flux at a separation of 0 "! 15 , or 0.04 times the stellar flux at 0 ". 50 . Cheetham et al. (2015) also did not find any companions down to 20 mas separation, at contrasts of 1-3 mag. With DSSI, we do not make any detections outward of 0 " 1 at $\Delta m \sim 4.4$ mag (flux contrast $\sim 0.02$ ) in the 692 or $880 \mathrm{~nm}$ bands.

The object is encircled by a class II disk, as reported by Evans et al. (2009). The disk was subsequently detected at 70, 160, 250, 350, and $500 \mu \mathrm{m}$ with Herschel by Rebollido et al. (2015), who tentatively classified it as transitional.

\section{A.12. EPIC 203935537/2MASS J16255615-2420481}

This star is also known as SR 4 and V2058 Oph. It has a long history of photometric and spectroscopic study, dating back to Struve \& Rudkjøbing's (1949) publication of emission line stars. Herbig \& Kameswara Rao (1972), Wilking et al. (1987), and Herbig \& Bell (1988) listed it in their catalogs of emission line stars. It has had a range of $\mathrm{H} \alpha$ EWs measured from 84 to $220 \AA$ as well as a low $v \sin i$ of $\sim 9 \mathrm{~km} \mathrm{~s}^{-1}$ (Bouvier 1990). Valenti et al. (1993) acquired blue spectra of this target, which revealed $\mathrm{H}(\beta, \gamma, \delta)$ and $\mathrm{Ca}$ in emission, as well as significant veiling at $4450 \AA$. Eisner et al. (2007) measured the veiling factor, $r$, to be $\sim 1.5$. Wilking et al. (2005) detected lithium absorption and $\mathrm{H} \alpha$ emission in this source. Reipurth et al. (1996) classified the $\mathrm{H} \alpha$ emission line profile as type IIR, in which there are two peaks of similar height. The accretion rate is estimated by Natta et al. (2006) to be a fairly high $10^{-6.74} M_{\odot} \mathrm{yr}^{-1}$, and the same authors detected $\mathrm{Pa} \beta$ in emission at an EW of $-19.0 \AA$. As suggested by Phelps \& Barsony (2004), the star may be the driver for a nearby Herbig Haro flow (HH 312) in the region.

The star is a ROSAT X-ray source. It is also an IRAS point source (Ichikawa \& Nishida 1989; Weintraub 1990; Clark 1991). Bontemps et al. (2001) also observed the class II disk with ISO. Andrews \& Williams (2007) detected the disk at $850 \mu \mathrm{m}$ with SCUBA, and they estimated a mass of $0.004 M_{\odot}$ based on SED fitting. Mohanty et al. (2013) used SCUBA-2 to measure a larger disk mass of $9.4 M_{\text {Jup }}\left(\sim 0.009 M_{\odot}\right)$. Spitzer $/$ IRS observations revealed a $10 \mu \mathrm{m}$ silicate feature, with a typical EW of $2.29 \mu \mathrm{m}$ (Furlan et al. 2009). Interferometric data and modeling led to an inferred inner disk radius of 0.112 au (Eisner et al. 2007). Pinte et al. (2008) estimated a very similar inner ring radius of 0.118 au from near-infrared interferometry. Andrews et al. (2010) observed the disk with the Submillimeter Array and found a centrally peaked morphology. Their modeling predicts an inner disk radius of $0.07 \mathrm{au}$ and an inclination of $39^{\circ}$; they infer an accretion rate of $10^{-6.8} M_{\odot} \mathrm{yr}^{-1}$, consistent with previous values. Millimeter and submillimeter ATCA observations by Ricci et al. (2010) led to an outer disk radius of $100-300$ au and a dust mass of $\sim 2 \times 10^{-5} M_{\odot}$. The disk is also detected with Herschel at 160 and $250 \mu \mathrm{m}$ (Rebollido et al. 2015).

The object is a known variable, as originally reported by Satyvaldiev (1972), Satyvoldiev (1982), and Kukarkin et al. (1977). It was followed up by Herbst et al. (1994), who found variations in the $V$ band of 12.73-12.93 during over 4000 days of monitoring. The amplitude was larger at blue wavelengths, with a typical $d U / d V$ of 2.4 mag. Shevchenko \& Herbst (1998) reported a $V$-band amplitude of $0.41 \mathrm{mag}$ over both short (hour-day) and long (years) timescales, with no detectable periodicity. Grankin et al. (2007) monitored the star for over seven years in the optical, finding a similar $V$-magnitude range of $12.60-13.09$.

EPIC 203935537/2MASS J16255615-2420481 is, to the best of our knowledge, a single star. Ghez et al.'s (1993) speckle imaging campaign did not reveal any companions down to 0 "! $1(0$ "! 2$)$, at a flux ratio of 17 (18). Nor did Simon et al.'s (1995) imaging survey, which was sensitive to separations of 0 ".005- 1 ". Our own DSSI observations did not show any companions down to 0 ". 1 separation at 4-5 mag of contrast (flux ratio $\sim 0.02$ ) in the 692 and $880 \mathrm{~nm}$ bands. Ratzka et al. (2005) searched for companions in high-resolution imaging but did not find any down to a flux ratio of 0.05 at a separation of 0 !" 15 . Neither Melo (2003) nor Guenther et al. (2007) detected any radial velocity variations indicative of spectroscopic binary status. Further high-resolution imaging (Richichi \& Percheron 2002; Richichi et al. 2005) failed to reveal companions.

\section{A.13. EPIC 203954898/2MASS J16263682-2415518}

This object was the subject of a multiplicity survey by Ratzka et al. (2005), but no companions were detected down to 0.05 (0.12) times the stellar brightness at $0.5(0$ " 15$)$ separation. Likewise, Duchêne et al. (2007) did not detect any companion, and reported that two faint stars observed at 6!" 1 and 6."3 separation (Duchêne et al. 2004) are likely background objects.

The star is a known X-ray emitter (Grosso et al. 2000; Imanishi et al. 2003; Gagné et al. 2004) and has a significant infrared excess, as indicated by ISO observations (Bontemps et al. 2001), Spitzer data (Cieza et al. 2009; Evans et al. 2009), and the AllWISE catalog (Cutri et al. 2013). The spectral index, $\alpha$, is 0.08 , indicating a flat disk (Evans et al. 2009).

A spectral type range of K7-M1 was reported by Erickson et al. (2011). Our own spectrum from the Palomar 200-inch telescope Double Spectrograph suggests K8. We adopt M0. Doppmann et al. (2005) report an effective temperature of $3700 \pm 56 \mathrm{~K}$, consistent with this spectral type. They also find a $v \sin i$ of $27 \pm 4.7 \mathrm{~km} \mathrm{~s}^{-1}$. The mass has been estimated to be $0.18 M_{\odot}$ (Natta et al. 2006), which is very low considering the temperature and youth of the object. We suspect that several groups have confused the source with a neighboring star, 2MASS J162637132415599, which is $\sim 9^{\prime \prime}$ away. For example, Wilking et al. (2005) list the object name ROXRA22, a ROSAT X-ray source at R.A. $=16: 26: 36.9$, decl. $=-24: 15: 53$ (Grosso et al. 2000). However, the Wilking et al. (2005) coordinates match the companion star at R.A. $=16: 26: 37.1$, decl. $=-24: 15: 59.9$, and the listed spectral type is later, at M5. The companion paper by Erickson et al. (2011) provides the same effective temperature, luminosity, and mass estimate, but an earlier spectral type range and lower extinction $\left(A_{V}=4.9\right)$. Because of these discrepancies, we derive our own spectral data, apart from the $v \sin i$ measurement. With the clear disk signatures, we can be confident that it is a young member of $\rho$ Ophiuchus. Natta et al. (2006) list an accretion rate of $<10^{-9.71} M_{\odot} \mathrm{yr}^{-1}$, based on near-infrared spectroscopy.

EPIC 203954898/2MASS J16263682-2415518 was monitored in the mid-infrared with Spitzer as part of the Young Stellar Object Variability project (YSOVAR; Rebull et al. 2014). Günther et al. (2014) obtained 81 data points spread 
over 34 days at $3.6 \mu \mathrm{m}$. The mean magnitude in this band was 8.15 , in line with previous brightness estimates (Cieza et al. 2009; Evans et al. 2009), and the standard deviation was 0.09 mag. The star showed variability at the $\sim 0.05$ mag level for the first 15 days of monitoring, followed by a $\sim 0.2 \mathrm{mag}$ increase followed by a similar decrease over the remaining 20 days of observation. WISE data also display a $\sim 0.3$ mag drop in the $W 1$ band from 2010 February to August.

\section{A.14. EPIC 204130613/2MASS J16145026-2332397}

This star, BV Sco, was listed as an irregular variable by Satyvoldiev (1982). It has been erroneously classified as an RR Lyrae star in SIMBAD; this type of variability is inconsistent with the stochasticity seen in our $K 2$ light curve. Like EPIC 204233955/2MASS J16072955-2308221, it was labeled by Lodieu et al. (2007) as a photometric non-member of Upper Scorpius (based on $Z Y J H K$ data), before it was re-classified as a strongly accreting member (Lodieu et al. 2011). It showed both $\mathrm{H} \alpha$ and $\mathrm{He} \mathrm{I}$ in emission $(-108$ and $-3.0 \AA \mathrm{EWs}$, respectively). Furthermore, they detected the calcium triplet lines and forbidden $\mathrm{O}$ I emission, suggesting outflows.

\section{A.15. EPIC 204226548/2MASS J15582981-2310077}

Also known as USco CTIO 33, Ardila et al. (2000) first identified this star as a candidate Upper Sco member. Preibisch et al. (2002) confirmed its youth via measurement of lithium absorption; they also detected strong $\mathrm{H} \alpha$ emission. With a spectral type of M3, it is estimated to be $0.36 M_{\odot}$ by Kraus \& Hillenbrand (2007). These authors also searched for spectroscopic and wide $\left(1^{\prime \prime}-30^{\prime \prime}\right)$ binary companions, but did not find any. This star was detected as a ROSAT X-ray source (RX J155829.5-231026) by Sciortino et al. (1998). Carpenter et al. (2006) were the first to detect an infrared excess, at 8 and $16 \mu \mathrm{m}$. Cieza et al. (2008) labeled it a transition disk source, with a mass of $<1.5 \times 10^{-3} M_{\odot}$ worth of material based on submillimeter data. No millimeter flux was detected by Mathews et al. (2012). Carpenter et al. (2014) report a submillimeter detection with ALMA, but the disk is unresolved. They constrain the dust mass to be $0.58 \pm 0.13 M_{\oplus}$. Dahm \& Carpenter (2009) measure significant $\mathrm{H} \alpha$ emission from a broad, flat-topped peak; their estimated accretion rate is $10^{-9.91} M_{\odot} \mathrm{yr}^{-1}$. Similar values were derived by Dahm (2010). Molecular gas is detected with Herschel/PACS (both $\mathrm{C}_{2} \mathrm{H}_{2}$ and $\mathrm{HCN}$ ) by Pascucci et al. (2013). Mathews et al. (2013) estimate less than $0.9 M_{\text {Jup }}$ worth of gas mass.

\section{A.16. EPIC 204233955/2MASS J16072955-2308221}

EPIC 204233955 is a spectral type M3 low-mass star in the Upper Scorpius association (Lodieu et al. 2011). It was initially classified as a photometric non-member by Lodieu et al. (2007), but Lodieu et al. (2011) found it to be an accreting source with strong emission lines, including $\mathrm{H} \alpha, \mathrm{He} \mathrm{I}$, and $\mathrm{O} \mathrm{I}$ forbidden lines. $\mathrm{H} \alpha$ and $\mathrm{He} \mathrm{I}$ EWs are $-150 \AA$ and $-3.0 \AA$, respectively. Mid-infrared data from the AllWISE survey (Cutri et al. 2013) reveal a significant infrared excess in all bands, indicative of a disk. Other than the work of Lodieu et al. (2007, 2011), this star has not been studied in detail.

\section{A.17. EPIC 204342099/2MASS J16153456-2242421}

EPIC 204342099, otherwise known as VV Sco, is a T Tauri star in the Upper Scorpius association. This object is also a known X-ray emitter from ROSAT observations (1RXS J161534.0-224218; Haakonsen \& Rutledge 2009) and XMM Newton observations (2XMM J161534.5-224241; Lin et al. 2012). Preibisch et al. (1998) obtained low- and mediumresolution spectra of this star as part of an X-ray selected sample of candidate Upper Scorpius members. They reported a spectral type of M1 and confirmed its youth via lithium absorption.

EPIC 204342099 is a known disk-bearing source, originally discovered with IRAS (Ichikawa \& Nishida 1989). It was studied in detail with Spitzer/IRS by Furlan et al. (2009), who list it under the id 16126-2235. They find a very strong $10 \mu \mathrm{m}$ silicate feature.

This star was first presented as a visual binary by GregorioHetem et al. (1992); the separation is $1{ }^{\prime \prime} 9$. Kraus \& Hillenbrand (2007) measured a spectral type of M3.5 for the companion. It is not clear whether this object is a co-moving Upper Scorpius member or a serendipitous field object; if the former, then the separation is 274 au. With DSSI speckle imaging, we measure the separation to be smaller at $1 . .50$, with a magnitude difference of $\Delta m=3.38$ at the $880 \mathrm{~nm}$ band. Kraus \& Hillenbrand (2008) also searched for closer companions with direct imaging and aperture masking, but did not find any within 240 mas, at a magnitude difference of 2.8 .

Sparse variability data is available for EPIC 204342099/ 2MASS J16153456-2242421. The star was first noted as variable by Petrov \& Satyvoldiev (1975). Batalha et al. (1998) monitored it for optical rotation signatures but did not detect any periodicities in the light curve.

Our HIRES spectrum suggests a spectral type of K9-M0 with lithium absorption present at strength $W_{\mathrm{Li}}=0.45 \AA$. The $\mathrm{H} \alpha$ emission is consistent with accretion (Table 1) and the profile exhibits a blueward asymmetry along with a redshifted absorption notch against the emission. Very weak and narrow profiles in $\mathrm{He}$ I, Fe II, and Ca II are also present in our data, along with very weak and narrow [O I].

\section{A.18. EPIC 204360807/2MASS J16215741-2238180}

There is no previous literature on this source. As with other objects under consideration here, there is significant veiling present with the spectral type changing from M2 in the bluer orders of our HIRES to possibly as late as M6 by about $8800 \AA$. Lithium has a strength of $W_{\mathrm{Li}}=0.27 A$. $\mathrm{H} \alpha$ emission as reported in Table 1 is very strong and there is He I, broad $\mathrm{NaD}$, and many $\mathrm{Fe}$ II lines. The $\mathrm{Ca}$ II triplet has multiple components and $\mathrm{O}$ I 8446 is present. Of the outflow lines only weak [O I] is seen.

With our DSSI speckle observations, we identify a companion at 0 " 48 separation with $\Delta m=0.74$ at $880 \mathrm{~nm}$.

\section{A.19. EPIC 204397408/2MASS J16081081-2229428}

This object was first identified as a candidate USco member based on proper motion by Lodieu et al. (2007) with Bouy \& Martín (2009) assigning it a membership probability of $99.9 \%$ based on the USNO-B proper motion. Slesnick et al. (2008) confirmed youth via variability and spectroscopy, assigning a spectral type of M5. They noted the star as an active accretor, based on a strong $\mathrm{H} \alpha$ emission line. Likewise, Lodieu et al. 
(2011) also spectroscopically confirmed this object as a USco member. Dahm et al. (2012) measured a radial velocity of about $-11 \mathrm{~km} \mathrm{~s}^{-1}$, slightly lower than the cluster mean, and a rotation velocity of $v \sin i=16.52 \pm 4.05$.

Riaz et al. (2012) analyzed WISE photometry for EPIC 204397408/2MASS J16081081-2229428, concluding that it harbors a class II disk. Luhman \& Mamajek (2012) also labeled it as a full disk.

\section{A.20. EPIC 204440603/2MASS J16142312-2219338}

This very low-mass star was classified by Lodieu et al. (2007) as a photometric and proper motion member of Upper Scorpius based on UKIDSS data. Following up with the AngloAustralian Telecope AAOmega spectrograph, Lodieu et al. (2011) obtained intermediate-resolution spectra of EPIC 204440603/2MASS J16142312-2219338 from 5750 to $8800 \AA$, deriving a spectral type of M5.75 and $\mathrm{H} \alpha \mathrm{EW}$ of $-94.5 \AA$. Their measured Na I and K I gravity-sensitive EWs as well as detection of $\mathrm{Li}$ I absorption cements the classification of this object as a young low-mass star.

\section{A.21. EPIC 204830786/2MASS J16075796-2040087}

This Upper Scorpius member was first identified as a strong $\mathrm{H} \alpha$ emission line star by The (1964). Kraus \& Hillenbrand (2009) obtained low-resolution spectra, which confirmed $\mathrm{H} \alpha$ emission at an EW of $-357 \AA$ and Ca II triplet emission as well. Detection of further emission lines (N II, S II, Fe II, Ni II, O I, and the Paschen series) suggested accretion-driven jets. These authors also associated EPIC 204830786/2MASS J160757962040087 with a wide-separation companion some $21 . " 5$ (3120 au) away. It has a significant infrared excess, as shown with IRAS (Carballo et al. 1992) and later with Spitzer and WISE by Luhman \& Mamajek (2012).

Our HIRES spectrum suggests a spectral type of late $G$ to early $\mathrm{K}$ but the spectrum is clearly heavily veiled; the lithium strength is $W_{\mathrm{Li}}=0.20 \AA$. Strong $\mathrm{H} \alpha$ emission is seen, as indicated in Table 1, with a blue-side absorption notch in the profile. Strong and broad He I, NaD, Fe II, Ca II, O I 8446, and perhaps other emission is present. Strong multi-component forbidden emission lines of [O I] and [S II] are seen, along with single-component [N II] and many [Fe II] lines.

Our DSSI speckle observations do not identify any companions outward of 0 !! 1 from this star, at a magnitude difference of $\Delta m \sim 4$ in the 692 and $880 \mathrm{~nm}$ bands.

\section{A.22. EPIC 204906020/2MASS J16070211-2019387}

Preibisch et al. (2001) detected EPIC 204906020 as a youthful member of the Upper Sco association via spectroscopic measurement of lithium absorption. This M5 star also has $\mathrm{H} \alpha$ emission, though it was weak enough in some observations to lead to a weak-lined T Tauri star classification. Kraus \& Hillenbrand (2007) reported the object to be an M5/ M5.5 wide binary with a 1". 63 separation. Kraus \& Hillenbrand (2009) found that the primary is itself a binary, with a 55 mas projected separation ( $8 \mathrm{au}$ at the distance of Upper Sco).

Carpenter et al. (2006) reported infrared excesses at 8 and $16 \mu \mathrm{m}$, indicating a disk. Carpenter et al. (2009) also detected an excess at $24 \mu \mathrm{m}$ with Spitzer/MIPS. Mathews et al. (2012) did not detect any cool dust around this system at millimeter wavelengths, at a $3 \sigma$ upper limit of $3.7 \times 10^{-3} M_{\text {Jup }}$. However, Mathews et al. (2013) used Herschel/PACS to detect
$6.6 \times 10^{-6} M_{\odot}$ worth of dust. Carpenter et al. (2014) detected but did not resolve the disk with ALMA.

EPIC 204906020/2MASS J16142312-2219338 is also known to have a circumstellar disk, as first reported by Riaz et al. (2012) and confirmed by Luhman \& Mamajek (2012). The SED slope is -1.3 , making it a class II disk (Riaz et al. 2012).

Our speckle observations with DSSI rule out any companions beyond 0 "! 1 at 4.6 mag contrast in the 692 and $880 \mathrm{~nm}$ bands.

\section{A.23. EPIC 204908189/2MASS J16111330-2019029}

This source appears in the literature only in the Luhman \& Mamajek (2012) WISE sample and the Barenfeld et al. (2016) ALMA study. Our HIRES spectrum suggests a spectral type of M1 with lithium absorption present at strength $W_{\mathrm{Li}}=0.15 \AA$. Strong $\mathrm{H} \alpha$ emission is seen, as indicated in Table 1, along with $\mathrm{He}$, weak but broad $\mathrm{NaD}$, weak and narrow Fe II, weak and narrow Ca II, but moderately broad O I 8446 emission. Weak and narrow $[\mathrm{O} \mathrm{I}]$ is also present.

\section{A.24. EPIC 205008727/2MASS J16193570-1950426}

There is no previous literature on this source. The HIRES spectrum is heavily veiled but appears to be a late $\mathrm{K}$ to $\mathrm{M} 3$ type, with lithium present at strength $W_{\mathrm{Li}}=0.55 \mathrm{~A} . \mathrm{H} \alpha$ emission as reported in Table 1 is strong and has multiple components. Additional emission includes $\mathrm{He}$, $\mathrm{NaD}, \mathrm{Fe}$ II, $\mathrm{Ca}$ II with the same profile shape as the $\mathrm{H} \alpha$ and $\mathrm{O}$ I 8446 is present. Outflow lines of [O I], [N II], and [S II] are also present, along with [Fe II].

\section{A.25. EPIC 205061092/2MASS J16145178-1935402}

There is no previous literature on this source. Our HIRES data indicate a spectral type of M5-M6 with lithium at $W_{\mathrm{Li}}=0.59 A$. Strong $\mathrm{H} \alpha$ emission, as indicated in Table 1 , is present along with $\mathrm{He}$ I, but no other emission lines.

\section{A.26. EPIC 205088645/2MASS J16111237-1927374}

Preibisch et al. (2002) first identified this star as an M5 member of the USco association, based on lithium absorption and broad H $\alpha$ emission (-50 A). Bouy \& Martín (2009) assigned it a membership probability of $99.9 \%$ based on the USNO-B proper motion. Martín et al. (2010) found a slightly later spectral type of M6 based on low-resolution spectra, and similarly broad $\mathrm{H} \alpha$ emission. The object displays an infrared excess confirmed by WISE to come from a full disk (Luhman \& Mamajek 2012).

\section{A.27. EPIC 205156547/2MASS J16121242-1907191}

There is no previous literature on this source. Our spectrum indicates a spectral type of M5-M6 with lithium at $W_{\mathrm{Li}}=0.56 A$. Moderate $\mathrm{H} \alpha$ emission, as indicated in Table 1 , is apparent with an asymmetric extension on the blue side of the profile. There is also He I but no other emission lines.

\section{Appendix B Spectral Energy Distributions}

We have computed spectral energy distributions for all 29 sources studied in this paper.

Photometry has been assembled from APASS (BV,gri), UKIDSS (ZY), 2MASS (JHK), and WISE (W1, W2, W3, W4). 

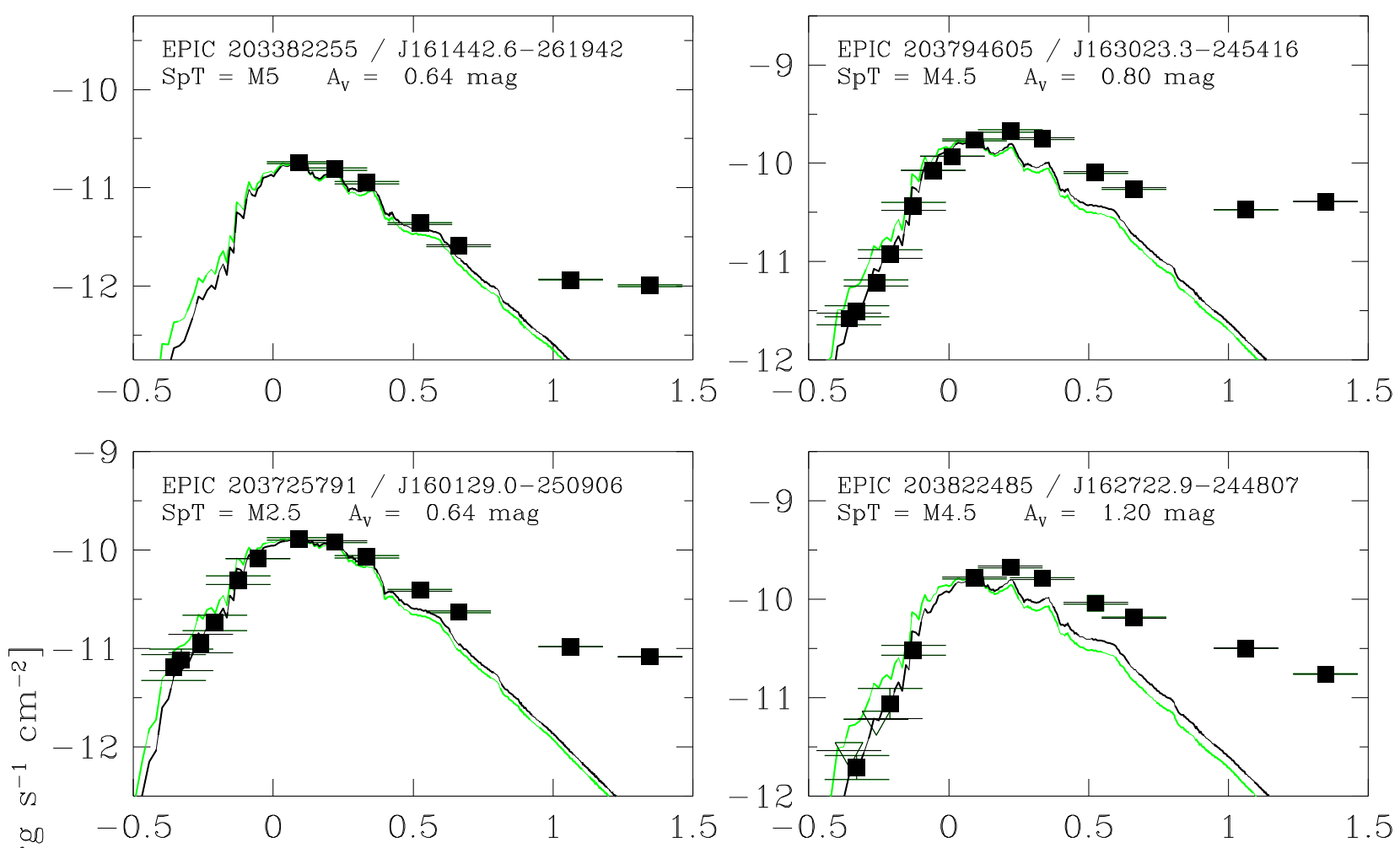

(1)
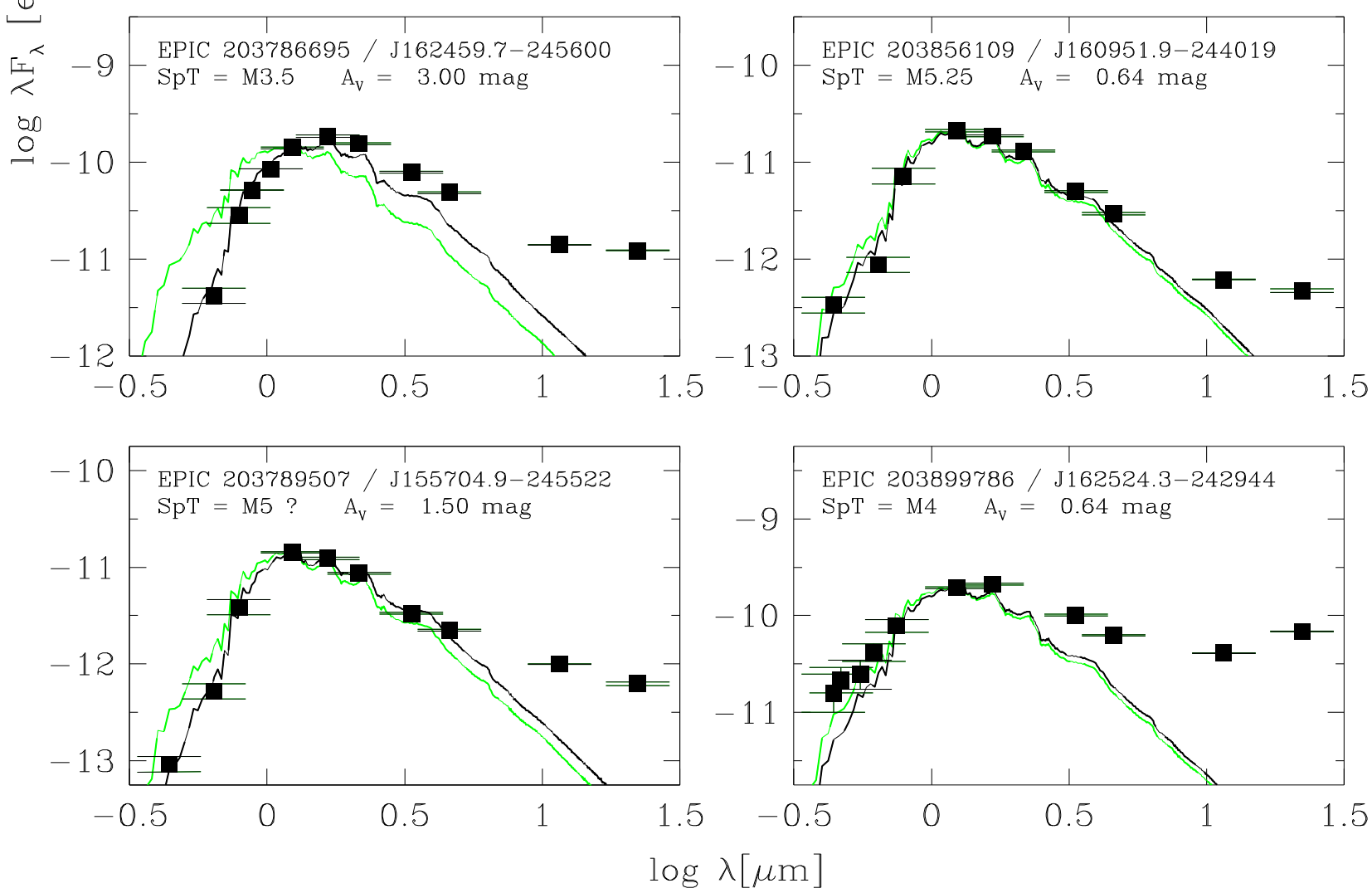

Figure 14. Spectral energy distributions for burster stars. Photometry has been assembled from APASS (BV, gri), UKIDSS (ZY), 2MASS (JHK), and WISE (W1, W2, W3, W4). The green line is a NextGen2 stellar atmosphere (Hauschildt et al. 1999) at $\log g=4.0$ and the temperature corresponding to the quoted spectral type. The black line is the same photosphere reddened by the quoted $A_{V}$. A value of 0.64 mag-the median $A_{V}$ we derive from assessment of extinction for several hundred members of the Upper Sco region—has been adopted as a minimum, with higher values of $A_{V}$ used when needed in order to fit the optical and near-infrared SED. (An extended version of this figure is available.) 


\section{References}

Aigrain, S., Parviainen, H., \& Pope, B. J. S. 2016, MNRAS, 459, 2408

Alencar, S. H. P., \& Batalha, C. 2002, ApJ, 571, 378

Alencar, S. H. P., Bouvier, J., Walter, F. M., et al. 2012, A\&A, 541, A116

Andre, P., \& Montmerle, T. 1994, ApJ, 420, 837

Andrews, S. M., \& Williams, J. P. 2007, ApJ, 671, 1800

Andrews, S. M., Wilner, D. J., Hughes, A. M., Qi, C., \& Dullemond, C. P. 2010, ApJ, 723, 1241

Antoniucci, S., Giannini, T., Li Causi, G., \& Lorenzetti, D. 2014, ApJ, 782, 51

Appenzeller, I., Krautter, J., \& Jankovics, I. 1983, A\&AS, 53, 291

Ardila, D., Martín, E., \& Basri, G. 2000, AJ, 120, 479

Aspin, C., Puxley, P. J., Hawarden, T. G., Paterson, M. J., \& Pickup, D. A. 1997, MNRAS, 284, 257

Aspin, C., \& Reipurth, B. 2009, AJ, 138, 1137

Audard, M., Ábrahám, P., Dunham, M. M., et al. 2014, in Protostars and Planets VI, ed. H. Beuther et al. (Tucson, AZ: Univ. Arizona Press), 387

Azevedo, R., Calvet, N., Hartmann, L., et al. 2006, A\&A, 456, 225

Barenfeld, S. A., Carpenter, J. M., Ricci, L., \& Isella, A. 2016, ApJ, 827, 142

Barsony, M., Koresko, C., \& Matthews, K. 2003, ApJ, 591, 1064

Batalha, C. C., Quast, G. R., Torres, C. A. O., et al. 1998, A\&AS, 128, 561

Bell, K. R., \& Lin, D. N. C. 1994, ApJ, 427, 987

Beristain, G., Edwards, S., \& Kwan, J. 2001, ApJ, 551, 1037

Blinova, A. A., Romanova, M. M., \& Lovelace, R. V. E. 2016, MNRAS, 459, 2354

Bontemps, S., André, P., Kaas, A. A., et al. 2001, A\&A, 372, 173

Bouvier, J. 1990, AJ, 99, 946

Bouvier, J., \& Appenzeller, I. 1992, A\&AS, 92, 481

Bouvier, J., Grankin, K., Ellerbroek, L. E., Bouy, H., \& Barrado, D. 2013, A\&A, 557, A77

Bouvier, J., Grankin, K. N., Alencar, S. H. P., et al. 2003, A\&A, 409, 169

Bouy, H., \& Martín, E. L. 2009, A\&A, 504, 981

Brandner, W., \& Zinnecker, H. 1997, A\&A, 321, 220

Carballo, R., Wesselius, P. R., \& Whittet, D. C. B. 1992, A\&A, 262, 106

Carpenter, J. M., Mamajek, E. E., Hillenbrand, L. A., \& Meyer, M. R. 2006, ApJL, 651, L49

Carpenter, J. M., Mamajek, E. E., Hillenbrand, L. A., \& Meyer, M. R. 2009, ApJ, 705, 1646

Carpenter, J. M., Ricci, L., \& Isella, A. 2014, ApJ, 787, 42

Casanova, S., Montmerle, T., Feigelson, E. D., \& Andre, P. 1995, ApJ, 439,752

Cheetham, A. C., Kraus, A. L., Ireland, M. J., et al. 2015, ApJ, 813, 83

Chou, M.-Y., Takami, M., Manset, N., et al. 2013, AJ, 145, 108

Cieza, L. A., Padgett, D. L., Allen, L. E., et al. 2009, ApJL, 696, L84

Cieza, L. A., Swift, J. J., Mathews, G. S., \& Williams, J. P. 2008, ApJ, 686,115

Clark, F. O. 1991, ApJS, 75, 611

Cody, A. M., Stauffer, J., Baglin, A., et al. 2014, AJ, 147, 82

Cohen, M., \& Kuhi, L. V. 1979, ApJS, 41, 743

Costigan, G., Vink, J. S., Scholz, A., Ray, T., \& Testi, L. 2014, MNRAS, 440,3444

Cutri, R. M., Wright, E. L., Conrow, T., et al. 2013, Explanatory Supplement to the AllWISE Data Release Products, Tech. Rep

Dahm, S. E. 2010, AJ, 140, 1444

Dahm, S. E., \& Carpenter, J. M. 2009, AJ, 137, 4024

Dahm, S. E., Slesnick, C. L., \& White, R. J. 2012, ApJ, 745, 56

D'Angelo, C. R., \& Spruit, H. C. 2010, MNRAS, 406, 1208

D’Angelo, C. R., \& Spruit, H. C. 2012, MNRAS, 420, 416

David, T. J., Hillenbrand, L. A., Cody, A. M., Carpenter, J. M., \& Howard, A. W. 2016, ApJ, 816, 21

Doppmann, G. W., Greene, T. P., Covey, K. R., \& Lada, C. J. 2005, AJ, 130,1145

Duchêne, G., Bontemps, S., Bouvier, J., et al. 2007, A\&A, 476, 229

Duchêne, G., Bouvier, J., Bontemps, S., André, P., \& Motte, F. 2004, A\&A, 427, 651

Eisner, J. A., Hillenbrand, L. A., White, R. J., et al. 2007, ApJ, 669, 1072

Erickson, K. L., Wilking, B. A., Meyer, M. R., Robinson, J. G., \& Stephenson, L. N. 2011, AJ, 142, 140

Evans, N. J., II, Dunham, M. M., Jørgensen, J. K., et al. 2009, ApJS, 181,321

Filin, A. Y., \& Satyvoldiev, V. 1975, PZ, 20, 161

Findeisen, K., Hillenbrand, L., Ofek, E., et al. 2013, ApJ, 768, 93

Furlan, E., Watson, D. M., McClure, M. K., et al. 2009, ApJ, 703, 1964

Gagné, M., Skinner, S. L., \& Daniel, K. J. 2004, ApJ, 613, 393

Ghez, A. M., Neugebauer, G., \& Matthews, K. 1993, AJ, 106, 2005

Goodson, A. P., \& Winglee, R. M. 1999, ApJ, 524, 159
Grankin, K. N., Melnikov, S. Y., Bouvier, J., Herbst, W., \& Shevchenko, V. S. 2007, A\&A, 461, 183

Green, J. D., Kraus, A. L., Rizzuto, A. C., et al. 2016, ApJ, 830, 29

Greene, T. P., \& Lada, C. J. 1996, AJ, 112, 2184

Gregorio-Hetem, J., Lepine, J. R. D., Quast, G. R., Torres, C. A. O., \& de La Reza, R. 1992, AJ, 103, 549

Grosso, N., Montmerle, T., Bontemps, S., André, P., \& Feigelson, E. D. 2000, A\&A, 359, 113

Guenther, E. W., Esposito, M., Mundt, R., et al. 2007, A\&A, 467, 1147

Günther, H. M., Cody, A. M., Covey, K. R., et al. 2014, AJ, 148, 122

Gutermuth, R. A., Megeath, S. T., Myers, P. C., et al. 2009, ApJS, 184, 18

Haakonsen, C. B., \& Rutledge, R. E. 2009, ApJS, 184, 138

Hartmann, L., Herczeg, G., \& Calvet, N. 2016, ARA\&A, 54, 135

Hartmann, L., Kenyon, S., \& Hartigan, P. 1993, in Protostars and Planets III, ed. E. H. Levy \& J. I. Lunine (Tucson, AZ: Univ. Arizona Press), 497

Hartmann, L., \& Kenyon, S. J. 1996, ARA\&A, 34, 207

Hauschildt, P. H., Allard, F., \& Baron, E. 1999, ApJ, 512, 377

Herbig, G. H., \& Bell, K. R. 1988, LicOB, 1111, 90

Herbig, G. H., \& Kameswara Rao, N. 1972, ApJ, 174, 401

Herbst, W., Herbst, D. K., Grossman, E. J., \& Weinstein, D. 1994, AJ, 108,1906

Herczeg, G. J., \& Hillenbrand, L. A. 2015, ApJ, 808, 23

Horch, E. P., Gomez, S. C., Sherry, W. H., et al. 2011, AJ, 141, 45

Horch, E. P., Howell, S. B., Everett, M. E., \& Ciardi, D. R. 2012, AJ, 144, 165

Howell, S. B., Everett, M. E., Sherry, W., Horch, E., \& Ciardi, D. R. 2011, AJ, 142,19

Howell, S. B., Sobeck, C., Haas, M., et al. 2014, PASP, 126, 398

Ichikawa, T., \& Nishida, M. 1989, AJ, 97, 1074

Imanishi, K., Nakajima, H., Tsujimoto, M., Koyama, K., \& Tsuboi, Y. 2003, PASJ, 55, 653

Joy, A. H. 1949, ApJ, 110, 424

Koresko, C. D. 2002, AJ, 124, 1082

Kraus, A. L., Cody, A. M., Covey, K. R., et al. 2015, ApJ, 807, 3

Kraus, A. L., \& Hillenbrand, L. A. 2007, ApJ, 662, 413

Kraus, A. L., \& Hillenbrand, L. A. 2008, ApJL, 686, L111

Kraus, A. L., \& Hillenbrand, L. A. 2009, ApJ, 703, 1511

Kraus, A. L., \& Hillenbrand, L. A. 2012, ApJ, 757, 141

Kukarkin, B. V., Kholopov, P. N., Fedorovich, V. P., et al. 1977, IBVS, 1248,1

Kulkarni, A. K., \& Romanova, M. M. 2008, MNRAS, 386, 673

Lii, P. S., Romanova, M. M., Ustyugova, G. V., Koldoba, A. V., \& Lovelace, R. V. E. 2014, MNRAS, 441, 86

Lin, D., Webb, N. A., \& Barret, D. 2012, ApJ, 756, 27

Lodato, G., \& Clarke, C. J. 2004, MNRAS, 353, 841

Lodieu, N. 2013, MNRAS, 431, 3222

Lodieu, N., Dobbie, P. D., \& Hambly, N. C. 2011, A\&A, 527, A24

Lodieu, N., Hambly, N. C., Jameson, R. F., et al. 2007, MNRAS, 374, 372

Lovelace, R. V. E., Romanova, M. M., \& Bisnovatyi-Kogan, G. S. 1995, MNRAS, 275, 244

Luhman, K. L., \& Mamajek, E. E. 2012, ApJ, 758, 31

Martin, E. L., Montmerle, T., Gregorio-Hetem, J., \& Casanova, S. 1998, MNRAS, 300, 733

Martín, E. L., Phan-Bao, N., Bessell, M., et al. 2010, A\&A, 517, A53

Mathews, G. S., Pinte, C., Duchêne, G., Williams, J. P., \& Ménard, F. 2013, A\&A, 558, A66

Mathews, G. S., Williams, J. P., Ménard, F., et al. 2012, ApJ, 745, 23

McCabe, C., Ghez, A. M., Prato, L., et al. 2006, ApJ, 636, 932

McClure, M. K., Furlan, E., Manoj, P., et al. 2010, ApJS, 188, 75

Melo, C. H. F. 2003, A\&A, 410, 269

Mohanty, S., Greaves, J., Mortlock, D., et al. 2013, ApJ, 773, 168

Montmerle, T., Koch-Miramond, L., Falgarone, E., \& Grindlay, J. E. 1983, ApJ, 269, 182

Najita, J. R., Andrews, S. M., \& Muzerolle, J. 2015, MNRAS, 450, 3559

Natta, A., Testi, L., Alcalá, J. M., et al. 2014, A\&A, 569, A5

Natta, A., Testi, L., \& Randich, S. 2006, A\&A, 452, 245

Ninan, J. P., Ojha, D. K., Baug, T., et al. 2015, ApJ, 815, 4

Parks, J. R., Plavchan, P., White, R. J., \& Gee, A. H. 2014, ApJS, 211, 3

Pascucci, I., Herczeg, G., Carr, J. S., \& Bruderer, S. 2013, ApJ, 779, 178

Pecaut, M. J., Mamajek, E. E., \& Bubar, E. J. 2012, ApJ, 746, 154

Petrov, P. P., \& Satyvoldiev, V. 1975, PZP, 2, 221

Phelps, R. L., \& Barsony, M. 2004, AJ, 127, 420

Pillitteri, I., Sciortino, S., Flaccomio, E., et al. 2010, A\&A, 519, A34

Pinte, C., Ménard, F., Berger, J. P., Benisty, M., \& Malbet, F. 2008, ApJL, 673, L63

Pontoppidan, K. M., Salyk, C., Blake, G. A., et al. 2010, ApJ, 720, 887

Prato, L. 2007, ApJ, 657, 338 
Preibisch, T., Brown, A. G. A., Bridges, T., Guenther, E., \& Zinnecker, H. 2002, AJ, 124, 404

Preibisch, T., Guenther, E., Zinnecker, H., et al. 1998, A\&A, 333, 619

Preibisch, T., Guenther, E., \& Zinnecker, H. 2001, AJ, 121, 1040

Ratzka, T., Köhler, R., \& Leinert, C. 2005, A\&A, 437, 611

Rebollido, I., Merín, B., Ribas, Á., et al. 2015, A\&A, 581, A30

Rebull, L. M., Cody, A. M., Covey, K. R., et al. 2014, AJ, 148, 92

Reipurth, B., \& Aspin, C. 2004, ApJL, 608, L65

Reipurth, B., Pedrosa, A., \& Lago, M. T. V. T. 1996, A\&AS, 120, 229

Reipurth, B., \& Zinnecker, H. 1993, A\&A, 278, 81

Riaz, B., Lodieu, N., Goodwin, S., Stamatellos, D., \& Thompson, M. 2012, MNRAS, 420, 2497

Ricci, L., Testi, L., Natta, A., \& Brooks, K. J. 2010, A\&A, 521, A66

Richichi, A., \& Percheron, I. 2002, A\&A, 386, 492

Richichi, A., Percheron, I., \& Khristoforova, M. 2005, A\&A, 431, 773

Ripepi, V., Balona, L., Catanzaro, G., et al. 2015, MNRAS, 454, 2606

Rizzuto, A. C., Ireland, M. J., \& Kraus, A. L. 2015, MNRAS, 448, 2737

Romanova, M. M., Kulkarni, A. K., \& Lovelace, R. V. E. 2008, ApJL, 673, L171

Romanova, M. M., Toropina, O. D., Toropin, Y. M., \& Lovelace, R. V. E. 2003a, ApJ, 588, 400

Romanova, M. M., Ustyugova, G. V., Koldoba, A. V., \& Lovelace, R. V. E. 2002, ApJ, 578, 420

Romanova, M. M., Ustyugova, G. V., Koldoba, A. V., \& Lovelace, R. V. E. 2004, ApJ, 610, 920

Romanova, M. M., Ustyugova, G. V., Koldoba, A. V., \& Lovelace, R. V. E. 2005, ApJL, 635, L165

Romanova, M. M., Ustyugova, G. V., Koldoba, A. V., \& Lovelace, R. V. E. 2011, MNRAS, 416, 416

Romanova, M. M., Ustyugova, G. V., Koldoba, A. V., Wick, J. V., \& Lovelace, R. V. E. 2003b, ApJ, 595, 1009
Rucinski, S. M., Matthews, J. M., Kuschnig, R., et al. 2008, MNRAS, 391, 1913

Rydgren, A. E. 1980, AJ, 85, 438

Rydgren, A. E., Strom, S. E., \& Strom, K. M. 1976, ApJS, 30, 307

Salyk, C., Pontoppidan, K. M., Blake, G. A., Najita, J. R., \& Carr, J. S. 2011, ApJ, 731, 130

Satyvaldiev, V. 1972, ATsir, 728, 5

Satyvoldiev, V. 1982, PZP, 4, 127

Schlafly, E. F., \& Finkbeiner, D. P. 2011, ApJ, 737, 103

Sciortino, S., Damiani, F., Favata, F., \& Micela, G. 1998, A\&A, 332, 825

Shevchenko, V. S., \& Herbst, W. 1998, AJ, 116, 1419

Simon, M., Ghez, A. M., Leinert, C., et al. 1995, ApJ, 443, 625

Slesnick, C. L., Hillenbrand, L. A., \& Carpenter, J. M. 2008, ApJ, 688, 377

Stauffer, J., Cody, A. M., Baglin, A., et al. 2014, AJ, 147, 83

Stauffer, J., Cody, A. M., Rebull, L., et al. 2016, AJ, 151, 60

Struve, O., \& Rudkjøbing, M. 1949, ApJ, 109, 92

The, P.-S. 1964, PASP, 76, 293

Valenti, J. A., Basri, G., \& Johns, C. M. 1993, AJ, 106, 2024

Vogt, S. S., Allen, S. L., Bigelow, B. C., et al. 1994, Proc. SPIE, 2198, 362

Vorobyov, E. I., \& Basu, S. 2015, ApJ, 805, 115

Weintraub, D. A. 1990, ApJS, 74, 575

White, R. J., \& Basri, G. 2003, ApJ, 582, 1109

Wilking, B. A., Lada, C. J., \& Young, E. T. 1989, ApJ, 340, 823

Wilking, B. A., Meyer, M. R., Robinson, J. G., \& Greene, T. P. 2005, AJ, 130,1733

Wilking, B. A., Schwartz, R. D., \& Blackwell, J. H. 1987, AJ, 94, 106

Zanni, C., \& Ferreira, J. 2013, A\&A, 550, A99

Zhu, Z., Hartmann, L., \& Gammie, C. 2009, ApJ, 694, 1045 